\title{
Transgenic Mouse Lines Subdivide Medial Vestibular Nucleus Neurons into Discrete, Neurochemically Distinct Populations
}

\author{
Martha W. Bagnall, ${ }^{1,3}$ Renna J. Stevens, ${ }^{1}$ and Sascha du Lac ${ }^{1,2,3}$ \\ ${ }^{1}$ Neuroscience Graduate Program, University of California, San Diego, La Jolla, California 92093, and ${ }^{2}$ Howard Hughes Medical Institute and ${ }^{3}$ The Salk \\ Institute for Biological Studies, La Jolla, California 92037
}

\begin{abstract}
The identification of neuron types within circuits is fundamental to understanding their relevance to behavior. In the vestibular nuclei, several classes of neurons have been defined in vivo on the basis of their activity during behavior, but it is unclear how those types correspond to neurons identified in slice preparations. By targeting recordings to neurons labeled in transgenic mouse lines, this study reveals that the continuous distribution of intrinsic parameters observed in medial vestibular nucleus (MVN) neurons can be neatly subdivided into two populations of neurons, one of which is GABAergic and the other of which is exclusively glycinergic or glutamatergic. In slice recordings, GABAergic neurons labeled in the EGFP (enhanced green fluorescent protein)-expressing inhibitory neuron (GIN) line displayed lower maximum firing rates $(<250 \mathrm{~Hz})$ than glycinergic and glutamatergic neurons labeled in the yellow fluorescent protein-16 (YFP-16) line (up to $500 \mathrm{~Hz}$ ). In contrast to cortical and hippocampal interneurons, GABAergic MVN neurons exhibited wider action potentials than glutamatergic (and glycinergic) neurons. Responses to current injection differed between the neurons labeled in the two lines, with GIN neurons modulating their firing rates over a smaller input range, adapting less during steady depolarization, and exhibiting less rebound firing than YFP-16 neurons. These results provide a scheme for robust classification of unidentified MVN neurons by their physiological properties. Finally, dye labeling in slices shows that both GABAergic and glycinergic neurons project to the contralateral vestibular nuclei, indicating that commissural inhibition is accomplished through at least two processing streams with differential input and output properties.
\end{abstract}

Key words: MVN; cerebellum; action potential; afterdepolarization; afterhyperpolarization; single-cell RT-PCR; vestibulo-ocular reflex

\section{Introduction}

The diversity of neuron types in the brain supports the complex processing that underlies fine-tuned behaviors. Identification and classification of these neuron types represents a critical step in understanding the behavioral relevance of their interconnections. In some regions of the brain, such as cortex and hippocampus, anatomy and morphology provide sufficient clues for broad cell-type classification. In other areas, including the brainstem and spinal cord, the lack of clear anatomical segregation of cell types has hampered progress in circuit analysis and, as a result, in pinpointing relationships between cellular properties and their consequences for behavior.

One well characterized behavior that relies on a comparatively simple circuit is the vestibulo-ocular reflex (VOR). The VOR acts

\footnotetext{
Received 0ct. 5, 2006; revised Dec. 10, 2006; accepted Dec. 30, 2006.

This work was supported by the Howard Hughes Medical Institute and National Institutes of Health Grant EY11027 (S.d.L.) and by a National Science Foundation graduate fellowship and a Salk Institute association grant (M.W.B.). We are grateful to I. van Welie for initial data on the GIN mice; L. Glickfeld for help with RT-PCR; K. Keener and B. Zing for excellent technical assistance; A. Nelson, M. Faulstich, and S. Moghadam for comparison data; S. M. Baca for discussion of action potential analysis; and Y. Yanagawa and K. Obata for the generous gift of GAD67-GFP mice.

Correspondence should be addressed to Sascha du Lac, Howard Hughes Medical Institute, The Salk Institute for Biological Studies, Systems Neurobiology Laboratories, 10010 North Torrey Pines Road, La Jolla, CA 92037. E-mail: sascha@salk.edu.

DOI:10.1523/JNEUROSCI.4322-06.2007

Copyright $\odot 2007$ Society for Neuroscience $\quad$ 0270-6474/07/272318-13\$15.00/0
}

to stabilize the retinal image despite head motion by transforming vestibular input into oculomotor output, so that the eyes rotate to counteract the effects of head movement. Decades of research into the VOR have yielded a wealth of information on its underlying circuitry, which consists of the brainstem vestibular nuclei, the cerebellum, and several oculomotor nuclei (Highstein and Holstein, 2005; Straka et al., 2005). The vestibular nuclei have been implicated as a site of VOR learning, just as their homologs, the deep cerebellar nuclei, have been implicated in eyeblink conditioning (Lisberger et al., 1994; du Lac et al., 1995; Mauk et al., 1998; Medina et al., 2000; Attwell et al., 2002; Ohyama et al., 2006). As a result, the VOR circuit provides a useful system for elucidating direct relationships between changes in behavior and their physiological and molecular underpinnings.

Interpretation of in vitro physiological experiments in the vestibular nuclei, however, has been limited by the absence of clear relationships to in vivo circuitry. In most studies, cell types have been identified in slice recording primarily on the basis of their intrinsic physiology, without reference to circuit function. Because the many cell types in the vestibular nuclei span a continuum of physiological properties (du Lac and Lisberger, 1995; Straka et al., 2005), the inability to identify them with certainty in slice preparations has restricted our understanding of the available forms of neuronal plasticity in the brainstem.

We sought better tools for associating the physiology of neu- 
Table 1. Primers for single-cell RT-PCR

\begin{tabular}{|c|c|c|c|c|}
\hline Product & $F / R$ & Sequence & Size (bp) & Reference \\
\hline \multirow{2}{*}{ VGluT1 (outer) } & $\mathrm{F}$ & GTCTTTGGCTTTGCCATTGT & \multirow[t]{2}{*}{996} & \multirow[t]{2}{*}{ Zhang et al., 2004} \\
\hline & $\mathrm{R}$ & CAGGGAGGCTATGAGGAACA & & \\
\hline \multirow[t]{2}{*}{ VGluT1 (inner) } & $\mathrm{F}$ & CACTATGGCTGTGTCATCTTCG & \multirow[t]{2}{*}{617} & \multirow[t]{2}{*}{ Zhang et al., 2004} \\
\hline & $\mathrm{R}$ & GTGGACATTATGTGACGACTGC & & \\
\hline \multirow[t]{2}{*}{ VGluT2 (outer) } & $\mathrm{F}$ & GAAATCAGCAAGGTTGGCAT & \multirow[t]{2}{*}{836} & \multirow[t]{2}{*}{ Zhang et al., 2004} \\
\hline & $\mathrm{R}$ & CAAGACTTGCTTGGTTGATATGTT & & \\
\hline \multirow[t]{2}{*}{ VGluT2 (inner) } & $\mathrm{F}$ & AGCAAGGTTGGCATGTTGTCTG & \multirow[t]{2}{*}{698} & \multirow[t]{2}{*}{ Zhang et al., 2004} \\
\hline & $\mathrm{R}$ & CGGTCCTTATAGGTGTACGCGT & & \\
\hline \multirow[t]{3}{*}{ GlyT2 } & F outer & CTTGGCTTGGGTGATTGTGTA & 587 & Ebihara et al., 2004 \\
\hline & Finner & CGGCCACTCAGATTTTCTTCT & \multirow[t]{2}{*}{381} & \multirow[t]{2}{*}{ Primer 3, NM148931 } \\
\hline & $\mathrm{R}$ & ACTTGGGAAACTCATCCGAGA & & \\
\hline \multirow[t]{3}{*}{ GAD67 } & Fouter & GATACTTGGTGTGGCGTAGCCC & 575 & Varju et al., 2002 \\
\hline & Finner & GCACAGAGACCGACTTCTCC & \multirow[t]{2}{*}{394} & \multirow[t]{2}{*}{ Primer 3, NM008077 } \\
\hline & $\mathrm{R}$ & ACGGGTGCAATTTCATATGTGAACATA & & \\
\hline \multirow[t]{3}{*}{ GAD65 } & Fouter & GGCGATGGAATCTTTTCTCCT & 1041 & Marsicano and Lutz, 1999 \\
\hline & Finner & CGCACTCTGGAAGACAATGA & \multirow[t]{2}{*}{205} & \multirow[t]{2}{*}{ Primer 3, NM008078 } \\
\hline & $\mathrm{R}$ & CGAGGCGTTCGATTTCTTCAA & & \\
\hline
\end{tabular}

VGluT1 and VGluT2 used nested primers, whereas the other products were seminested. F/R, Forward/reverse primers.

rons recorded in slice preparations with their role in the VOR circuit. The mouse offers many advantages in genetic approaches to this problem, and its behavioral performance and adaptive capability in the VOR are excellent (De Zeeuw et al., 1998; Stahl et al., 2000; Faulstich et al., 2004). In this study, two lines of transgenic mice, EGFP (enhanced green fluorescent protein)expressing inhibitory neurons (GIN) and yellow fluorescent protein-16 (YFP-16), are used to elucidate the intrinsic physiology of neurochemically delimited sets of neurons in the medial vestibular nucleus (MVN), without the need for extensive postrecording processing (Feng et al., 2000; Oliva et al., 2000). In addition, we show a simple method for identifying commissurally projecting neurons in standard slices. Finally, we target identified commissural neurons in slice recordings to show that glycinergic and GABAergic commissurally projecting neurons in the MVN have distinctive intrinsic physiologies. The results provide a classification scheme of MVN neurons grounded in a molecular rather than a phenomenological framework.

\section{Materials and Methods}

Mouse lines and morphological analysis. GABAergic neurons were identified in GIN mice, which express EGFP in a subset of glutamic acid decarboxylase 67 (GAD67)-positive neurons (Oliva et al., 2000). To find a mouse line with a fluorescently labeled population distinct from that of GIN neurons, we examined mice generated by insertion of a modified thyl promoter driving enhanced YFP (EYFP) (Feng et al., 2000). In the YFP-16 line, EYFP expression in large neurons in the deep cerebellar nuclei (M. W. Bagnall and S. du Lac, unpublished observations) suggested its potential utility in the homologous vestibular nuclei. We also used the GAD67-GFP line of mice, in which GFP is knocked in to the native Gad1 locus (Tamamaki et al., 2003), to target GABAergic neurons for recording.

One GIN and one YFP-16 animal were perfused transcardially with PBS followed by $4 \%$ paraformaldehyde. After removal of the brain and postfixation for $\sim 30 \mathrm{~min}$, the brain was sunk in $30 \%$ sucrose and sectioned at $30 \mu \mathrm{m}$ on a freezing microtome. From each mouse line, the unilateral MVN from two sections, at approximately bregma -5.88 and -6.24 , was imaged with a $100 \times$ objective on an Olympus FV300 confocal microscope (Olympus, Tokyo, Japan) in $0.4 \mu \mathrm{m}$ steps. Neurons were included for morphological analysis if the entire soma was enclosed within the resulting z-stack.

Slice preparation and electrophysiology. Coronal slices of the brainstem $(250-300 \mu \mathrm{m})$ were prepared with a DSK-1500E or Leica VT1000S Vibratome. Slices were incubated in carbogenated artificial CSF (ACSF) at $34^{\circ} \mathrm{C}$ for $30 \mathrm{~min}$ and then at room temperature for $>30 \mathrm{~min}$. ACSF contained (in mm) $124 \mathrm{NaCl}, 26 \mathrm{NaHCO}_{3}, 5$ $\mathrm{KCl}, 1.3 \mathrm{MgCl}_{2}, 2.5 \mathrm{CaCl}_{2}, 1 \mathrm{NaH}_{2} \mathrm{PO}_{4}$, and 11 dextrose. For recording, slices from 17- to 28d-old (P17-P28) (mean P21 \pm 3 SD) YFP-16 and GIN mice were submerged in a recording chamber and perfused with $34^{\circ} \mathrm{C}$ carbogenated ACSF. An additional dataset from recordings made at room temperature from animals of similar age (P16-P37; mean P25 \pm 5 ) is included for comparison in Table 2.

Fluorescence was imaged with a fluorescein isothiocyanate filter and Optiquip illumination on an Olympus microscope with a $40 \times$ objective. MVN neurons labeled in the YFP-16 line display a wide range of intensities; dim neurons were not targeted for recording. Neurons were visualized under infrared illumination with differential interference contrast optics. Wholecell current-clamp recordings were made with an AxoClamp 2B or a MultiClamp 700B amplifier. Data were filtered at $10 \mathrm{kHz}$, digitized with an ITC- 16 board at $40 \mathrm{kHz}$ for action potential analysis and at $20 \mathrm{kHz}$ for all other measurements, and collected in Macintosh OS 9 with a custom-written code in Igor Pro 4. Electrodes were filled with an internal solution containing (in mM) $140 \mathrm{~K}$ gluconate, $10 \mathrm{HEPES}, 8 \mathrm{NaCl}$, 0.1 EGTA, $2 \mathrm{MgATP}, 0.3$ $\mathrm{Na}_{2} \mathrm{GTP}$, and, in some cells, $0.1 \%$ biocytin (Sigma, St. Louis, MO).

For commissural studies, 450-500 $\mu \mathrm{m}$ slices were made from P12P17 animals. Afterward, slices were temporarily removed from ACSF and placed on Whatman filter paper. A 27 gauge needle was used to poke several holes in the MVN on one side of the brainstem, particularly in the midventral region, which is the primary target of commissural fibers (Ladpli and Brodal, 1968; Epema et al., 1988). Crystallized fluorolabeled dextran was delivered by needle into the MVN of GIN (fluororuby) or YFP-16 (Texas Red; both 10,000 molecular weight; Invitrogen, Eugene, OR) slices. Slices were rinsed in ACSF, removed from filter paper, and returned to the incubation chamber. After $6-8 \mathrm{~h}$ incubation at $\sim 32^{\circ} \mathrm{C}$, slices were either fixed in $4 \%$ paraformaldehyde for histology or used for electrophysiological recordings as above. Commissural electrophysiology was performed on slices from $\mathrm{P} 14-\mathrm{P} 17$ animals.

Reverse transcriptase-PCR. After neuronal physiology was recorded, negative pressure was applied as the pipette was removed from the slice. The electrode contents were expelled with pressurized air, and deoxyribonucleotide triphosphates $(0.5 \mu \mathrm{l} ; 10 \mathrm{~mm})$ and random hexamer primers $(0.5 \mu \mathrm{l} ; 500 \mathrm{ng})$ were added. After $5 \mathrm{~min}$ incubation at $65^{\circ} \mathrm{C}$, samples were kept on ice until the addition of reverse transcriptase (RT) buffer, Superscript III reverse transcriptase, and dithiothreitol (all from Invitrogen). Reactions were then incubated at $50^{\circ} \mathrm{C}$ for $1 \mathrm{~h}$ and $75^{\circ} \mathrm{C}$ for $10 \mathrm{~min}$ and stored at $4^{\circ} \mathrm{C}$. Positive controls [whole-brain RNA preparations (RNeasy kit; Qiagen, Hilden, Germany)] and negative controls (recording pipettes dipped in the slice and removed without attaching to a cell) were included in every round of RT-PCR.

Primers and sources are listed in Table 1. Primers were selected so that the primer or the product spanned an exon-intron boundary to eliminate the possibility of genomic contamination. For the first PCR, primers to five gene products were multiplexed: vesicular glutamate transporter 1 (VGluT1), VGluT2, glycine transporter 2 (GlyT2), GAD65, and GAD67. In some cases, actin primers were included as well (data not shown). The entire volume of the RT reaction $(\sim 10 \mu \mathrm{l})$ was mixed with water, buffer, and $0.5 \mu \mathrm{l} \mathrm{Taq}$ (Eppendorf, Westbury, NY) and heated to $94^{\circ} \mathrm{C}$ for $2 \mathrm{~min}$. Primers were added to the mixture for a final concentration of $\sim 0.2 \mu \mathrm{M}$ in a final reaction volume of $100 \mu \mathrm{l}$, and PCR continued for 20 cycles of $1 \mathrm{~min}$ at $94^{\circ} \mathrm{C}, 1 \mathrm{~min}$ at $56^{\circ} \mathrm{C}$, and $1 \mathrm{~min}$ at $72^{\circ} \mathrm{C}$. For the second PCR, 2 $\mu \mathrm{l}$ of the first PCR was mixed with $50 \mu \mathrm{M}$ deoxyribonucleotide triphosphates, buffer, $0.5 \mu \mathrm{l} \mathrm{Taq}$, and $0.2 \mu \mathrm{M}$ primers for a single gene product (usually nested or seminested; in some instances, the same primer set was used for the first and second PCRs) (Table 1). The PCR protocol was the same as above but for 35 cycles. Approximately $10 \mu \mathrm{l}$ of this reaction product was run on a $1.5 \%$ agarose gel with Tris-acetate-EDTA. 
Physiological analysis. Recordings were targeted primarily to the rostral two-thirds of the MVN in both magnocellular and parvocellular divisions, approximately bregma -5.88 to -6.48 . Neurons were included in this study only if they were capable of firing continuously during a $1 \mathrm{~s}$ depolarization and if their spike height was $>50 \mathrm{mV}$ ( $45 \mathrm{mV}$ for commissural studies). Analysis was performed as in Sekirnjak and du Lac (2006). For action potential analysis, cells were injected with hyperpolarizing or depolarizing current (if required) to force a rate of 8-12 spikes per second for $1-5 \mathrm{~s}$. These spikes were averaged together by alignment at their peaks. Spike threshold was defined as the $V_{\mathrm{m}}$ at which the voltage derivative reached $10 \mathrm{~V} / \mathrm{s}$. Spike width was measured at threshold, and spike half-width was measured at the halfway point between threshold and peak. The maximum afterhyperpolarization (AHP) was defined as the difference between threshold and the most negative $V_{\mathrm{m}}$ reached during the AHP; the time to maximum AHP was defined as the time difference between this point and the spike peak. The afterdepolarization (ADP) was quantified as the maximum value of the spike derivative during the $1 \mathrm{~ms}$ after the spike repolarized to threshold value.

To examine how neurons respond to various levels of inputs, steps of depolarizing current ( $1 \mathrm{~s}$ ) were delivered in increasing amplitude until the neuron was unable to sustain action potentials across the whole step. Firing rate was taken as the average of the reciprocal of interspike intervals across the entire step. In current steps that yielded a firing rate of $35-45 \mathrm{~Hz}$ at the end of the step, firing rate adaptation was measured as the ratio of the firing rate over $100 \mathrm{~ms}$ at the end of the step versus the beginning of the step. The first $50 \mathrm{~ms}$ of firing was excluded from analysis (Sekirnjak and du Lac, 2006). Cellular gain was measured as the slope of the best-fit line to a graph of firing rate versus current step amplitude. Input resistance was measured at approximately $-75 \mathrm{mV}$ by injecting a small negative current step ( -5 to $-50 \mathrm{pA}$ ) to produce a $2-3 \mathrm{mV}$ hyperpolarization. Six repetitions were averaged for analysis.

A dataset from similar-aged wild-type mice (P21-P25) that had been sham-operated $3 \mathrm{~d}$ previously for vestibular labyrinthectomy was analyzed for comparison (A. B. Nelson, M. Faulstich, and S. Moghadam, unpublished data). All data were analyzed with custom-written code in Igor Pro 4, and statistical tests were performed in KaleidaGraph 3.6. Significance was determined by Student's unpaired $t$ test and is reported as mean $\pm \mathrm{SD}$, except as noted.

\section{Results}

Two transgenic lines of mice label different subsets of neurons in the MVN. In the YFP-16 line (Feng et al., 2000), a modified thy1 promoter drives EYFP expression; in the GIN line (Oliva et al., 2000), a portion of the Gad1 promoter (gene product, GAD67) drives EGFP expression. The gross distribution of EYFP+ (hereafter, YFP-16) and EGFP+ (hereafter, GIN) neurons in fixed tissue sections of the MVN was approximately complementary. YFP-16 neurons were located throughout the rostrocaudal extent of the MVN, with particularly heavy distribution in the more lateral, magnocellular regions (Fig. $1 A$ ). In contrast, although GIN neurons were also found throughout the MVN, they were most concentrated in parvocellular areas close to the fourth ventricle, with a smaller scattering concentrated more laterally in the magnocellular region (Fig. $1 B$ ). Thus the territories of the two cell types are dissimilar but overlapping, in keeping with reports of intermingled cell-type distribution in the MVN (Serafin et al., 1991; Sekirnjak and du Lac, 2002, 2006; Takazawa et al., 2004).

Fluorescent neurons of both lines had heterogeneous morphology (Fig. 1C,D). Analysis with confocal microscopy of neurons in similar slices from each line (YFP-16, $n=108$; GIN, $n=$ 149) showed that YFP-16 neurons on average measured $17.5 \pm$ $4.3 \mu \mathrm{m}$ on the long axis and $9.2 \pm 2.7 \mu \mathrm{m}$ on the short axis, whereas GIN neurons were significantly smaller, with measurements of $14.9 \pm 3.6$ and $8.1 \pm 2.1 \mu \mathrm{m}$ (long axis, $p<0.0001$; short axis, $p<0.001)$. The approximate somatic area, quantified as the product of long and short axes, was thus significantly larger
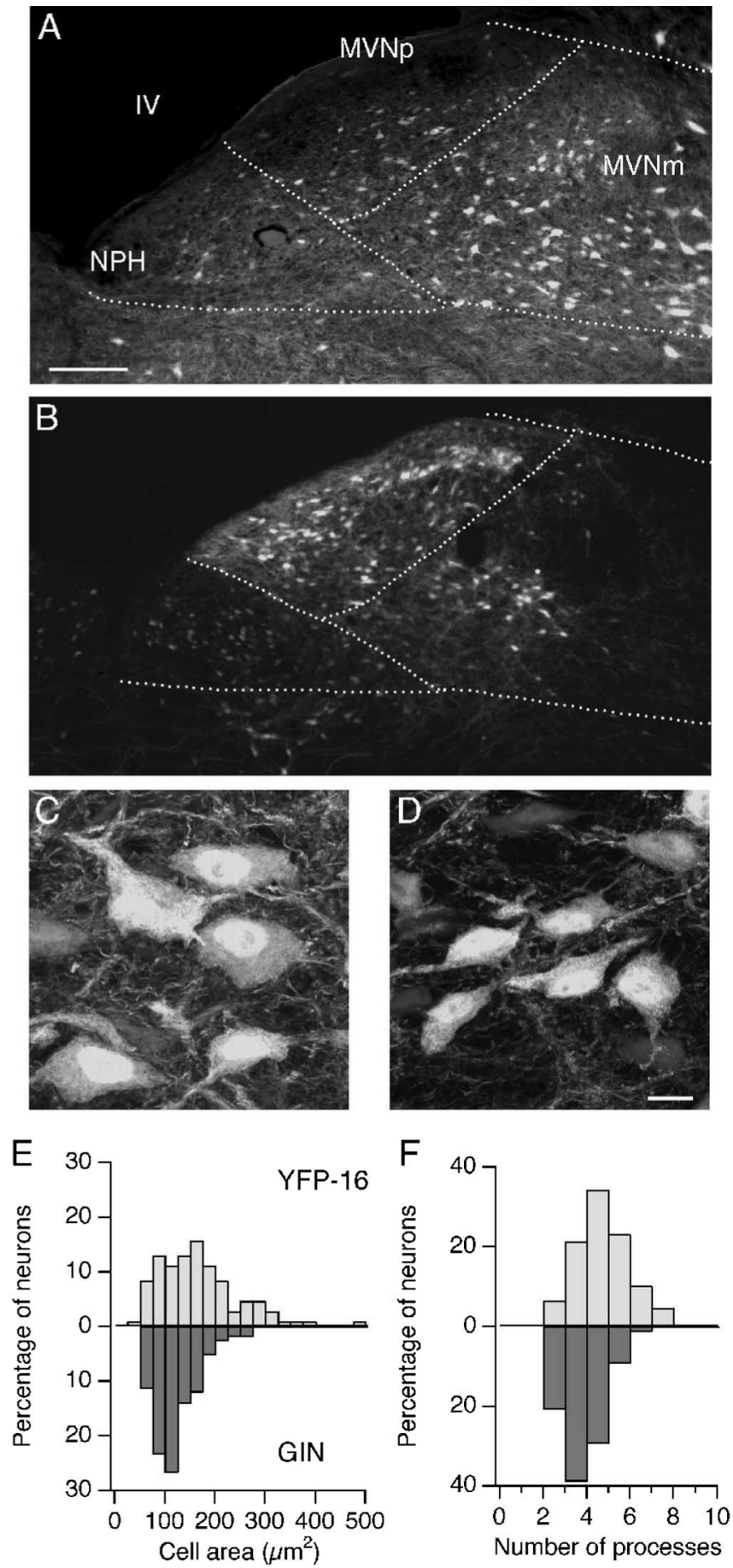

Figure 1. Distribution and morphology of YFP-16 and GIN neurons in the MVN. Coronal sections are from the YFP-16 ( $\boldsymbol{A})$ and GIN ( $\boldsymbol{B})$ lines. MVNp, Parvocellular MVN; MVNm, magnocellular MVN; NPH, nucleus prepositus hypoglossi; IV, fourth ventricle (darkened for contrast). Scale bar: $\boldsymbol{A}$ (for $\boldsymbol{A}, \boldsymbol{B}), 200 \mu \mathrm{m}$. $\boldsymbol{C}, \boldsymbol{D}$, Magnified view of YFP-16 neurons ( $\boldsymbol{C}$ ) and GIN neurons in MVN (D). Scale bar: $\boldsymbol{D}$ (for $\boldsymbol{C}, \boldsymbol{D}), 10 \mu \mathrm{m}$. $\boldsymbol{E}$, Histogram of soma size, measured as the product of length and width. YFP-16 neurons are significantly larger than GIN neurons ( $p<0.001)$. $\boldsymbol{F}$, Histogram of number of processes extending from cell body. On average, YFP-16 neurons extend significantly more primary dendrites than GIN neurons $(p<0.0001) . n=108$ (YFP-16) and 160 (GIN) neurons for both graphs.

in YFP-16 than in GIN neurons (Fig. $1 E)(p<0.0001)$. Most YFP-16 neurons (94\%) were multipolar and typically extended three to five primary dendrites from the soma (average, $4.2 \pm$ 1.2 ); in contrast, $\sim 20 \%$ of GIN neurons were bipolar, and on 

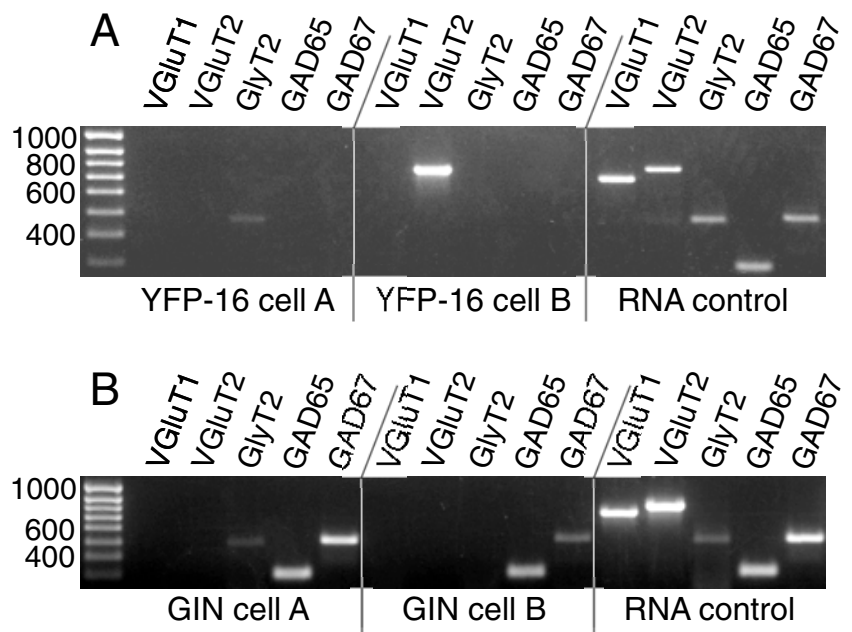

Figure 2. Single-cell RT-PCR indicates that YFP-16 neurons are glutamatergic or glycinergic, whereas GIN neurons are GABAergic. RT-PCR was performed on YFP-16 and GIN neurons from slice recordings. Neurons were assayed for five indicators of transmitter content: VGluT1, VGluT2, GlyT2, GAD65, and GAD67. Two neurons are shown from each line. A, YFP-16 neuron A is VGluT2 +, whereas neuron B is GlyT2 + . B, GIN neuron A expressed the GABAergic markers GAD67 and GAD65, as well as the glycinergic marker GlyT2; GIN neuron B expressed only GAD67 and GAD65. Right, RNA controls were $0.5 \mathrm{ng}$ RNA extracts from whole mouse brain processed alongside single cells.

average they extended only $3.3 \pm 1.0$ processes (Fig. $1 F)(p<$ $0.0001)$. Despite these significant differences in size and morphology, the two populations displayed a large degree of overlap (Fig. $1 E, F$ ), precluding simple anatomical identification in slice.

YFP-16 and GIN neurons express different neurotransmitters GIN neurons comprise a subset of GABAergic cells (Oliva et al., 2000), but the transmitter phenotype of the majority of YFP-16 neurons is unknown [with the exception of motor neurons, all of which are labeled (Walsh and Lichtman, 2003)]. Antibody staining against protein markers of the transmitter phenotype is complicated by the fact that most such proteins are only weakly expressed somatically; therefore, we turned to RT-PCR. An RTPCR analysis of transmitter phenotype of single neurons targeted for recording revealed that $50 \%$ ( 9 of 18) of YFP-16 neurons were positive for GlyT2, which is found in glycinergic neurons (Poyatos et al., 1997), whereas the remaining 50\% were positive for VGluT2, which labels glutamatergic neurons (Fig. 2A) (Fremeau et al., 2001). Notably, no YFP-16 neurons were found to be positive for either GAD67 ( 0 of 16) or GAD65 ( 0 of 11), the two major isoforms of GAD, nor primarily for VGluT1 (1 of 16), a VGluT most commonly found in excitatory neurons with low probabilities of release (Fig. 2A) (Fremeau et al., 2004).

Having shown that YFP-16 neurons are glutamatergic or glycinergic, but not GABAergic, we verified the transmitter phenotype of GIN neurons by the same method. RT-PCR analysis indicated that GIN neurons in the MVN expressed GAD67 (23 of 28 ) and GAD65 (26 of 28) but did not express VGluT1 or VGluT2 (0 of 28), in contrast to YFP-16 neurons (Fig. 2B). In keeping with reports of colocalization of GABAergic and glycinergic markers in a subset of MVN neurons (Tanaka and Ezure, 2004), we found that $50 \%$ (14 of 28) of GIN neurons also expressed GlyT2 (Fig. 2 B). Therefore, GIN and YFP-16 neurons represent neurochemically distinct populations within the MVN.

\section{Intrinsic physiology}

MVN neurons are physiologically diverse with respect to their responses to current injection and the shape of their action po- tentials (Serafin et al., 1991; Johnston et al., 1994); however, because neurons of similar physiology may be found in quite different regions of the vestibular nuclei, whereas neurons of dissimilar physiology may lie right next to each other, there has been a dearth of effective strategies for targeting particular subclasses of neurons in slice recordings. Because the GIN and YFP-16 lines label two distinct populations of neurons in the MVN, they provide excellent tools for identifying the physiology of GABAergic and non-GABAergic neurons. We targeted GIN and YFP-16 neurons for physiological recording in whole-cell patch clamp in the $\mathrm{MVN}$, primarily in mid-rostral regions (bregma -5.88 to -6.48). Recordings were made at $34^{\circ} \mathrm{C}$ in juvenile (P17-P28; average, P21 \pm 3 ) mice from 59 (GIN) and 49 (YFP-16) neurons. YFP-16 neurons vary widely in brightness; recordings were targeted to the most visible, and therefore brightest, neurons. We analyzed action potential shape, which has been used in several laboratories to classify MVN neurons into two or three groups (Serafin et al., 1991; Johnston et al., 1994). In addition, we characterized the response of the cells to depolarizing and hyperpolarizing current steps. A dataset of 61 cells recorded in separate experiments from wild-type animals (P21-P25) was included in analyses to show how the physiological characteristics of YFP-16 and GIN neurons compare with those of unidentified neurons.

\section{Action potential waveform is largely distinct between YFP-16} and GIN neurons

Fewer YFP-16 than GIN neurons fired spontaneously ( 22 of 49 vs 47 of 59; $p<0.001$; Fisher's exact test), but within spontaneously active neurons, YFP-16 neurons fired at higher spontaneous rates than GIN neurons [YFP-16, $24.5 \pm 15.5 \mathrm{~Hz}$ (range, 3-71 Hz); GIN, $16.3 \pm 11.1 \mathrm{~Hz}$ (range, $1-60 \mathrm{~Hz}$ ); $p<0.05$ ]. No difference was seen in the regularity of spontaneous action potentials, as measured by coefficient of variation (YFP-16, $0.14 \pm 0.17$; GIN, $0.11 \pm 0.13 ; p>0.4)$. Examples of typical action potentials recorded in YFP-16 and GIN neurons are shown in Figure 3, $A$ and $B$. As is characteristic of MVN neurons, these examples exhibit remarkably swift rising and falling phases of the action potential, with the total spike width usually $<1 \mathrm{~ms}$. The AHP in some instances is monophasic (Fig. $3 B$, top) and in others displays a fast and a slow component. Three differences are salient between the YFP-16 and GIN neuron spikes at the top of the panels: the width of the action potential, the magnitude of the AHP, and the presence or absence of an ADP dividing the AHP into an early and late component. Although these examples are distinctive, some action potentials from each population bear strong resemblances to each other (Fig. $3 A, B$, examples in bottom half).

To facilitate action potential comparisons across neurons, direct current was applied to force a firing rate of $\sim 10 \mathrm{~Hz}$, and the resulting spikes were averaged for analysis. Because MVN neurons have previously been subdivided on the basis of the presence of one or two components to the AHP (Serafin et al., 1991; Johnston et al., 1994; Beraneck et al., 2003), the ADP, which separates these components, was analyzed (see Materials and Methods). When the ADP was plotted versus action potential half-width (a slightly more robust measure than action potential width because it is less sensitive to threshold definition), YFP-16 and GIN populations were largely distinct on the resulting continuum (Fig. 3C). The ADP was significantly larger and the action potential half-width significantly narrower in the YFP-16 than in the GIN neurons (Table 2). This correlation may reflect either that the currents necessary to produce both a rapid repolarization and an ADP are coregulated (MacLean et al., 2003) or simply that the ADP is evident only in cells with an action potential that 
A
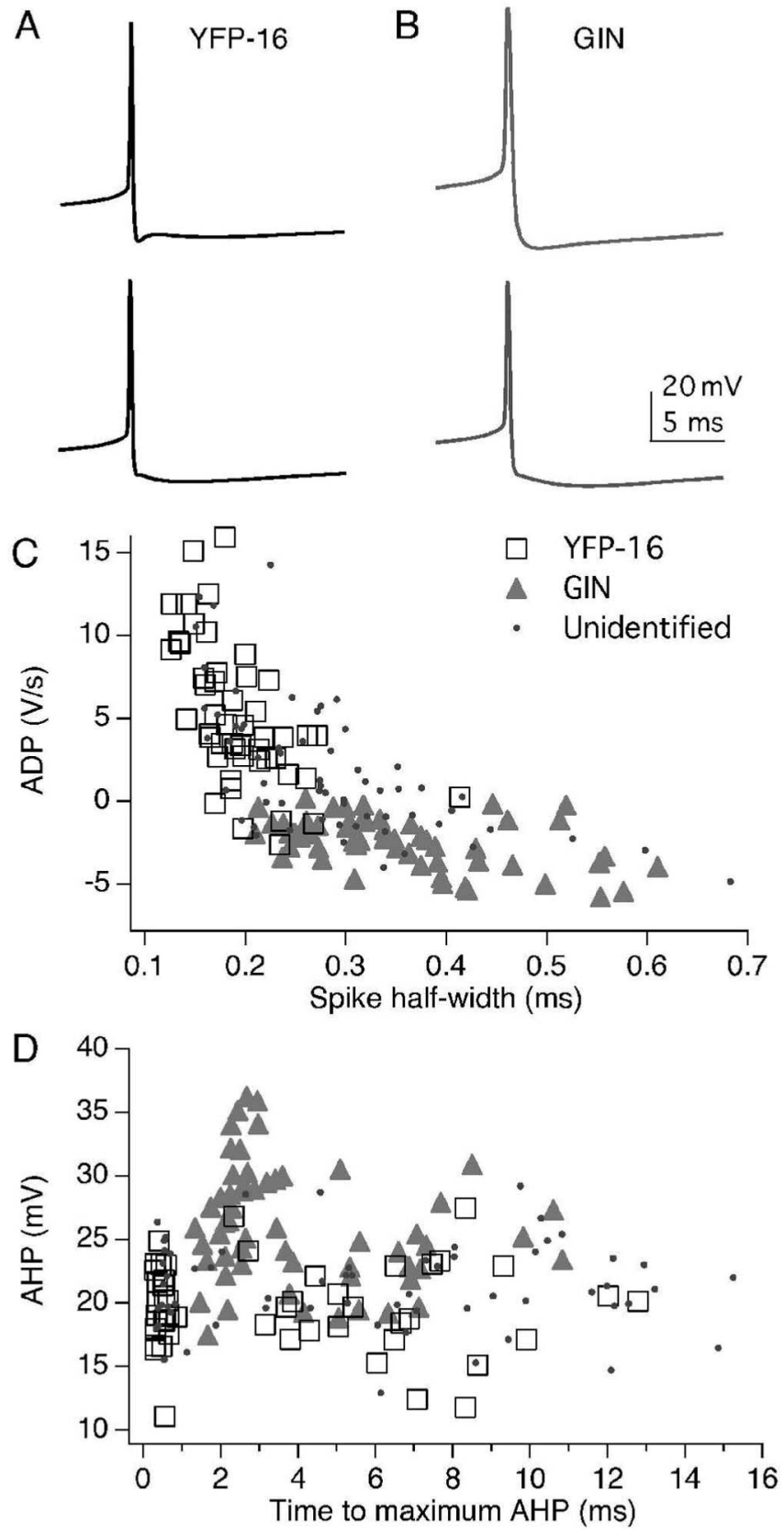

Figure 3. Action potentials differ between YFP-16 and GIN neurons. $A$, Example action potentials from two YFP-16 neurons (black). $\boldsymbol{B}$, Examples from two GIN neurons (gray). C, Relationship between ADP after spike repolarization and action potential width at half-height. Each point represents one neuron [YFP-16 (open squares), $n=49$; GIN (gray triangles), $n=59$; unidentified neurons from a separate study (dark gray dots), $n=61]$. D, Maximum depth of the AHP in YFP-16 and GIN neurons plotted versus the time after the spike peak when that maximum is reached. Symbols and $n$ are the same as those in $C$.

repolarizes swiftly. The ADP has been attributed, variously, to calcium channels (Autret et al., 2005; Metz et al., 2005), to persistent sodium channels (Yue et al., 2005), or to a contribution of current from multiple dendrites (Quadroni and Knopfel, 1994). Given that YFP-16 neurons extend significantly more dendrites than GIN neurons (Fig. $1 F$ ), our data are consistent with the last of those possibilities.

Previous studies have distinguished MVN cell types by examining the magnitude of the AHP (with respect to threshold) in relation to the time at which the maximum is reached (Takazawa et al., 2004; Sekirnjak and du Lac, 2006). YFP-16 neurons fell primarily into two groups, based on whether the AHP reached a maximum before or after the ADP $(<1 \mathrm{~ms}$ or $>3 \mathrm{~ms}$ after the spike) (Fig. 3D). Similarly, some GIN neurons repolarized swiftly (1-3 ms after spike), whereas others reached a maximum AHP $>4 \mathrm{~ms}$ after the action potential (Fig. 3D). Notably, however, no GIN neurons reached the deepest portion of the AHP as quickly as the earliest YFP-16 neurons, despite the fact that a few GIN neurons have spike widths that are comparable to those of YFP-16 neurons (Fig. 3C). Furthermore, GIN neurons on average exhibit significantly deeper maximum AHPs than do YFP-16 neurons (Fig. 3C, Table 2), an effect attributable mostly to those neurons that repolarize in the $2-4 \mathrm{~ms}$ after the action potential (a time when very few YFP-16 neurons reach a maximum AHP). The larger magnitude of the AHP relative to spike threshold derives from two sources: first, that GIN neurons exhibit higher spike thresholds $(-45.3 \pm 4.4 \mathrm{mV})$ than YFP-16 neurons $(-50.7 \pm 4.0 \mathrm{mV} ; p<0.0001)$, accounting for $5.4 \mathrm{mV}$ of the total $6.6 \mathrm{mV}$ average AHP difference between the groups. The remaining $1.2 \mathrm{mV}$ difference in average AHP magnitude is likely attributable to differences in the amplitude of repolarizing currents between YFP-16 and GIN neurons (A. Gittis and S. du Lac, unpublished observations). In all measures of action potential waveform, GIN and YFP-16 neurons span the same extent as unidentified neurons recorded in wild-type animals (Fig. 3C,D, dark gray dots), suggesting that the two populations together represent a high proportion of MVN neurons typically targeted for recording.

\section{YFP-16 and GIN neurons display different signal transformation properties}

Input-output characteristics are widely heterogeneous across MVN neurons (Serafin et al., 1991; Ris et al., 2001; Sekirnjak and du Lac, 2002). To explore the dynamic range of GABAergic and non-GABAergic neurons in the MVN, we applied depolarizing steps of current for $1 \mathrm{~s}$, increasing the current on each step until the neuron was unable to sustain firing across the step. Figure 4 shows the resulting firing patterns in a typical YFP-16 (Fig. 4A) and GIN (Fig. $4 B$ ) neuron. The YFP-16 neuron attained instantaneous firing rates of more than 500 spikes per second, declining over the course of the current step to $\sim 300$ spikes per second, whereas the highest firing rate that the GIN neuron could sustain was only $\sim 200$ spikes per second. The maximum firing rate, an indication of the output range of a neuron, was taken as the average of the firing rate on the highest step for which the neuron fired without fail. Maximum firing rates of YFP-16 neurons were significantly higher than those of GIN neurons, whereas their input resistances were lower (Fig. 4C, Table 2), in keeping with previous reports of an inverse relationship between these measures (Sekirnjak and du Lac, 2002). Comparison with data from wild-type animals revealed that the YFP-16 and GIN populations together span the same values of maximum firing rate and input resistance as unidentified neurons (Fig. 4C). The amplitude of the largest current step, a measure of the functional input range over which the neuron could sustain firing, was on average about three times larger in YFP-16 than GIN neurons (Fig. 4D). Together, these data indicate that YFP-16 neurons have a wide dynamic range of inputs and outputs, whereas GIN neurons are constrained to a narrower operating range.

The transformation of current input to firing rate output occurs not only over a wider range in YFP-16 than in GIN neurons, but also with different temporal properties. Firing rate declines appreciably over the course of the current step in the YFP-16 
Table 2. Intrinsic characteristics of YFP-16 and GIN neurons in the MVN are distinct at both physiological and room temperatures

\begin{tabular}{|c|c|c|c|c|c|c|c|}
\hline & \multicolumn{2}{|c|}{ Physiological temperature $\left(34^{\circ} \mathrm{C}\right)$} & \multicolumn{2}{|l|}{$n$} & \multirow[b]{2}{*}{$p$ value } & \multicolumn{2}{|c|}{ Room temperature $\left(23^{\circ} \mathrm{C}\right)$} \\
\hline & YFP-16 & GIN & YFP-16 & GIN & & YFP-16 & GIN \\
\hline AP width (ms) & $0.52 \pm 0.11$ & $0.82 \pm 0.20$ & 49 & 59 & $<0.0001$ & $0.68 \pm 0.10$ & $0.96 \pm 0.18$ \\
\hline AP half-width (ms) & $0.19 \pm 0.05$ & $0.36 \pm 0.10$ & 49 & 59 & $<0.0001$ & $0.28 \pm 0.05$ & $0.42 \pm 0.09$ \\
\hline $\mathrm{AHP}(\mathrm{mV})$ & $19.6 \pm 3.4$ & $26.2 \pm 4.7$ & 49 & 59 & $<0.0001$ & $19.2 \pm 3.0$ & $23.3 \pm 3.0$ \\
\hline Maximum derivation (V/s) & $521 \pm 102$ & $345 \pm 84$ & 49 & 59 & $<0.0001$ & $450 \pm 101$ & $328 \pm 76$ \\
\hline Minimum derivation (V/s) & $444 \pm 117$ & $224 \pm 69$ & 49 & 59 & $<0.0001$ & $340 \pm 92$ & $202 \pm 47$ \\
\hline $\operatorname{ADP}(V / s)$ & $5.14 \pm 4.28$ & $-2.48 \pm 1.56$ & 49 & 59 & $<0.0001$ & $5.85 \pm 2.99$ & $-0.96 \pm 1.22$ \\
\hline Threshold (mV) & $-50.7 \pm 4.0$ & $-45.3 \pm 4.4$ & 49 & 50 & $<0.0001$ & $-48.6 \pm 5.5$ & $-47.1 \pm 3.6$ \\
\hline Input resistance $(\mathrm{M} \Omega$ ) & $107 \pm 92$ & $269 \pm 137$ & 44 & 51 & $<0.0001$ & $114 \pm 76^{a}$ & $400 \pm 180^{a}$ \\
\hline Maximum firing rate $(\mathrm{Hz})$ & $353 \pm 121$ & $154 \pm 44$ & 40 & 43 & $<0.0001$ & $307 \pm 84$ & $118 \pm 26$ \\
\hline Adaptation ratio $(40 \mathrm{~Hz})$ & $0.81 \pm 0.13$ & $0.91 \pm 0.09$ & 48 & 44 & $<0.0001$ & $0.82 \pm 0.10$ & $0.93 \pm 0.08$ \\
\hline Gain < $80 \mathrm{~Hz}(\mathrm{~Hz} / \mathrm{nA})$ & $242 \pm 117$ & $242 \pm 122$ & 48 & 49 & 0.996 & $217 \pm 81$ & $268 \pm 134$ \\
\hline Gain > $80 \mathrm{~Hz}(\mathrm{~Hz} / \mathrm{nA})$ & $158 \pm 84$ & $206 \pm 93$ & 46 & 46 & 0.011 & $134 \pm 75$ & $203 \pm 103$ \\
\hline Gain/input resistance & $1.90 \pm 0.85$ & $0.81 \pm 0.38$ & 42 & 41 & $<0.0001$ & $1.32 \pm 0.56$ & $0.59 \pm 0.36$ \\
\hline Rebound firing $(\mathrm{Hz})$ & $42 \pm 40$ & $12 \pm 13$ & 38 & 24 & $<0.0001$ & $53 \pm 61$ & $\mathrm{n} / \mathrm{a}$ \\
\hline
\end{tabular}

Room temperature recordings were as follows: for spike parameters, $n=26$ (YFP-16) and 37 (GIN); for cell parameters, $n=14-37$. With the exception of threshold and gain $<80 \mathrm{~Hz}$, all parameters are significantly different between YFP-16 and $\operatorname{GIN}$ at $23^{\circ} \mathrm{C}(p<0.05)$. Values indicate mean \pm SD.

${ }^{a}$ Some input resistance measurements were made in voltage clamp rather than current clamp.
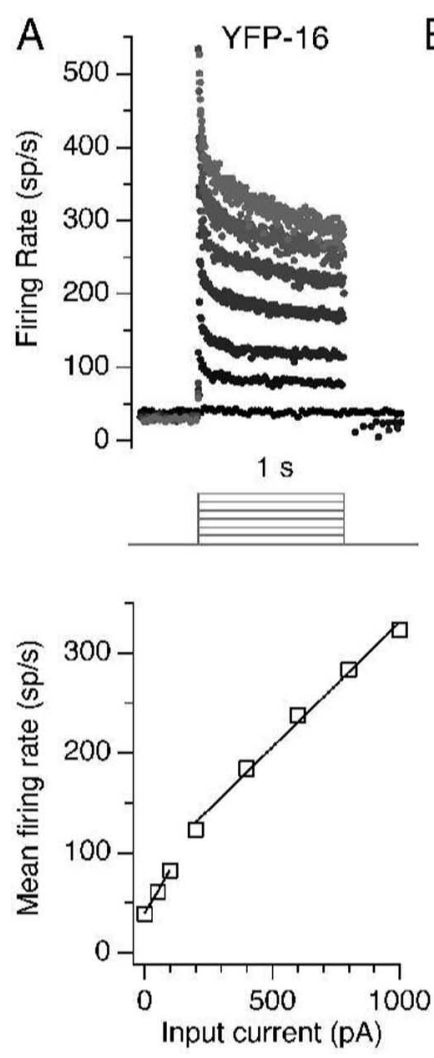
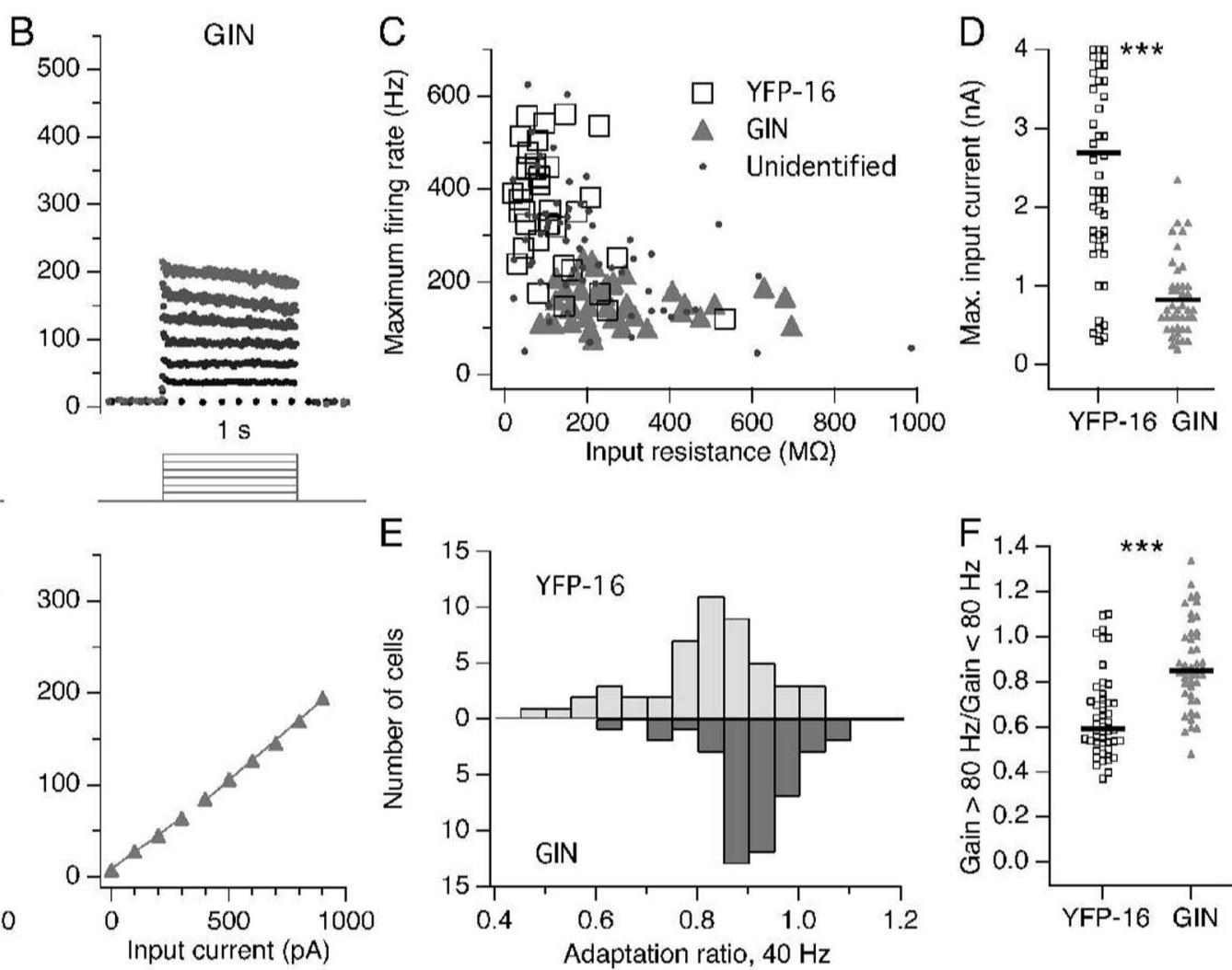

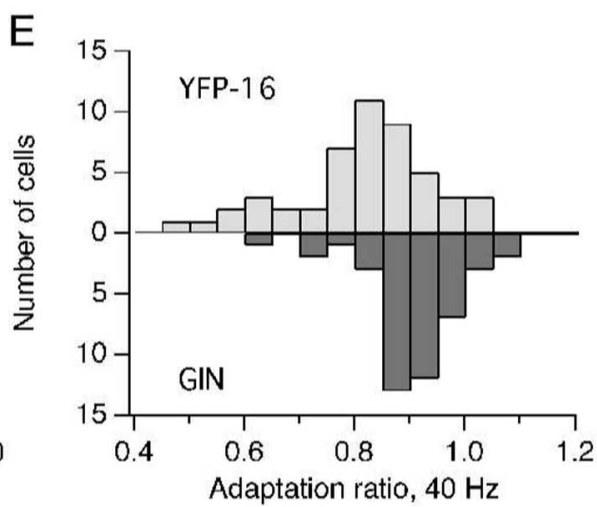

Figure 4. Input- ouput properties in YFP-16 and GIN neurons. Shown are examples of responses to depolarizing current steps in a YFP-16 neuron ( $\boldsymbol{A}$ ) and a GIN neuron ( $\boldsymbol{B}$ ). Steps of increasing amplitude were given until the neuron could no longer fire action potentials throughout the whole step. Bottom panels, Mean firing rate evoked during depolarization is plotted versus the amplitude of the current step for each neuron shown above. The break in line fit indicates the measurement of gain below and above $80 \mathrm{~Hz}$ for each neuron. $\boldsymbol{C}$, Scatterplot of maximum firing rate (average over whole step) versus input resistance measured below spike threshold. Each point represents one neuron (YFP-16, $n=40 ;$ GIN, $n=43$; unidentified neurons, $n=61$ ). D, Maximum input current for which a neuron could sustain action potentials during $1 \mathrm{~s}$ of depolarization. YFP-16 neurons can respond to approximately threefold more current than GIN neurons ( $p<0.0001$ ) (Table 2 ). Bars indicate means. Three YFP-16 neurons whose maximum input current exceeded $4 \mathrm{nA}$ are not shown here for graphical clarity. $\boldsymbol{E}$, Histogram of adaptation ratio, defined as the firing rate at the end of the $1 \mathrm{~s}$ depolarization divided by firing rate at the beginning. YFP-16 neurons adapted significantly more than GIN neurons $(p<0.0001)$ (Table 2$)$. $\boldsymbol{F}$, Ratio of cellular gain in high range ( $>80$ spikes per second) to gain in low range ( $<80$ spikes per second). GIN neurons were significantly more linear than YFP-16 neurons ( $p<0.0001)$ (Table 2).

neuron shown in Figure $4 A$, but less so in the GIN neuron (Fig. $4 B)$. The adaptation ratio, defined as the firing rate at the end divided by the firing rate at the beginning of the $1 \mathrm{~s}$ step (see Materials and Methods), was significantly higher in GIN neurons $(0.91 \pm 0.09)$ than in YFP-16 neurons $(0.81 \pm 0.13 ; p<0.0001)$, indicating less adaptation across the step in GABAergic than in
non-GABAergic cells. These results are plotted in histogram form in Figure $4 E$.

The current-firing rate relationship is linear in many MVN neurons (Fig. $4 A, B$, bottom) and can be described by a single gain value. In some cells, a modest bilinearity can be discerned when comparing the gain below $\sim 80 \mathrm{~Hz}$ or the gain above (the 
two line fits in each example neuron). Overall, YFP-16 and GIN neurons had comparable levels of excitability, whether gain was measured at firing rates below $80 \mathrm{~Hz}$ or above (Table 2); however, when comparing gain above $80 \mathrm{~Hz}$ with gain below, YFP-16 neurons were significantly less linear (ratio of high range gain to low range gain; $0.65 \pm 0.19)$ than GIN neurons $(0.88 \pm 0.19 ; p<$ 0.0001) (Fig. 4F). Furthermore, cellular excitability above spike threshold (gain) was not closely related to cellular excitability below spike threshold (input resistance). In YFP-16 neurons, the ratio of gain to input resistance was more than twice as high as in GIN neurons (Table 2).

After a $1 \mathrm{~s}$ hyperpolarizing current step of $\sim-30 \mathrm{mV}$, MVN neurons tend to fire at elevated rates compared with those before the step, a phenomenon termed "rebound firing" (Fig. 5A). High rates of rebound firing are associated with low input resistance and strong adaptation (Sekirnjak and du Lac, 2002). Furthermore, neurons with extensive innervation from the floccular lobe of the cerebellum (floccular target neurons), identified in slice in an L7GFP transgenic mouse, have rebound firing ranging from 80 to $250 \mathrm{~Hz}$, whereas non-floccular target neurons usually display rebound firing of $0-100 \mathrm{~Hz}$ (Sekirnjak et al., 2003; Sekirnjak and du Lac, 2006). We found that YFP-16 neurons had significantly higher rebound than GIN neurons, in agreement with predictions from previous work (Sekirnjak and du Lac, 2002) (Fig. $5 B$ ). Comparison of rebound firing with the ADP magnitude revealed that the measures are highly correlated (Fig. $5 C)\left(R^{2}=\right.$ $0.61 ; p<0.0001)$. The correlation might arise because the same current underlies both characteristics [e.g., calcium, which has been shown to be related to rebound bursts in the deep cerebellar nuclei (Molineux et al., 2006)], or because of a coregulation of the currents responsible for the characteristics. The many differences between the intrinsic physiology of the YFP-16 and GIN cell groups likely reflect a different distribution of ionic currents in the two cell types, particularly those activated above spike threshold (Gittis and du Lac, unpublished observations).

Subsets of both YFP-16 and GIN neurons expressed the glycine marker GlyT2, but the presence or absence of GlyT2 did not distinguish cell types in either line (Table 3). Glycinergic YFP-16 neurons displayed physiological characteristics similar to glutamatergic YFP-16 neurons, and GIN neurons expressing GlyT2 were similar to GIN neurons that did not express GlyT2. In contrast, the intrinsic physiological properties of GlyT2-expressing GIN neurons differed substantially from those of GlyT2expressing YFP-16 neurons (Table 3).

\section{Both YFP-16 and GIN neurons project commissurally}

GABAergic, glycinergic, and glutamatergic neurons make commissural projections between the two halves of the MVN (Shimazu and Precht, 1966; Kasahara et al., 1968; Mano et al., 1968; Gacek, 1978; Dieringer and Precht, 1979a,b; Spencer et al., 1989; Holstein et al., 1999). Commissural inhibition sets up the push-pull circuitry that underlies the bilaterality of the VOR and is thought to support recovery of the VOR after labyrinthectomy or canal plug (Bienhold and Flohr, 1978; Galiana et al., 1984; Furuya et al., 1992) (but see Smith et al., 1986). We hypothesized that the differences in physiology between YFP-16 and GIN neurons might reflect their circuit roles: perhaps one sends projections contralaterally, whereas the other does not. Because of the topology of the vestibular nucleus, however, it is rare to be able to trace axons of cells recorded in slice preparations for substantial distances. As a result, neuronal fills are frequently uninformative in cell-type identification. We turned instead to dye labeling of axon tracts (Sekirnjak and du Lac, 2006).
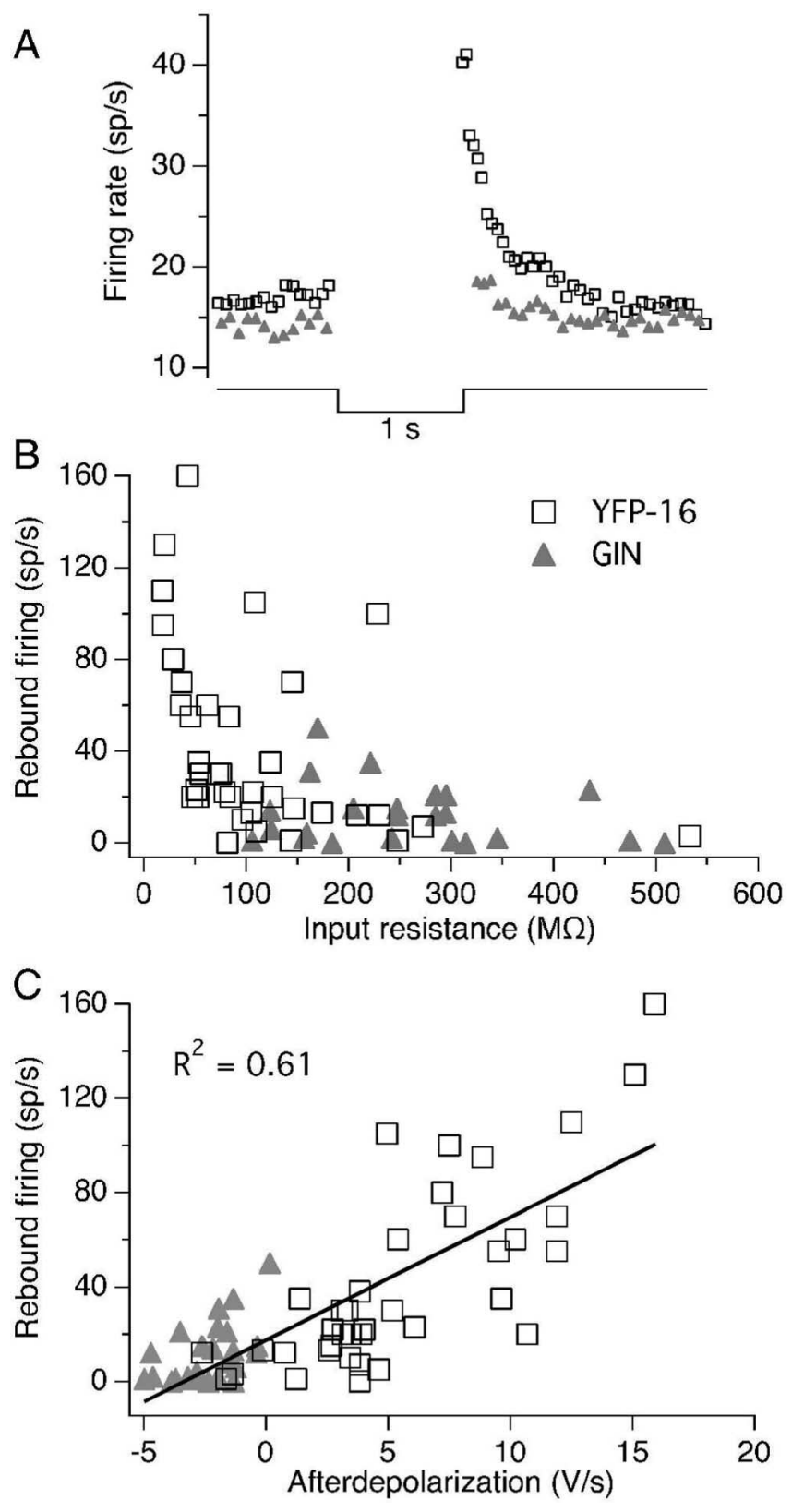

Figure 5. Post-inhibitory rebound firing in YFP-16 and GIN neurons. $A$, Examples of rebound firing after $1 \mathrm{~s}$ of hyperpolarizing current injection in YFP-16 (open squares) and GIN (closed triangles) neurons. Neurons were forced to fire $10-20 \mathrm{~Hz}$ and hyperpolarized $\sim 30 \mathrm{mV}$ to facilitate comparisons across cells. $\boldsymbol{B}$, Data from individual neurons are plotted against their input resistance. YFP-16 neurons exhibit significantly more rebound firing than GIN neurons, although there is large heterogeneity (Table 2). Each symbol represents one neuron (YFP-16, $n=38 ; G I N, n=24)$. C, Rebound firing and ADP are positively correlated across all neurons ( $n$ as in $\boldsymbol{B})$. One neurons with rebound of 300 spikes per second was excluded from analyses. $\mathrm{sp} / \mathrm{s}$, Spikes per second.

To determine whether any of the YFP-16 or GIN neurons project contralaterally, thick slices were made from young (P12P17) animals, and crystals of lipophilic dye [fluororuby (GIN mice) or Texas Red (YFP-16) mice] were deposited into one side of the MVN, typically throughout the ventrolateral aspect. Fluorescently labeled neurons were seen contralateral to the site of injection 6-8 h after injections, suggesting that commissural axons had transported the dye retrogradely to cell bodies.

To verify that neurons were making commissural connections, we first examined the midline of the slice. Figure $6 B$ shows 
Table 3. Glutamatergic and glycinergic YFP-16 neurons exhibit physiology similar to each other but not to glycinergic GIN neurons

\begin{tabular}{|c|c|c|c|c|}
\hline & \multicolumn{2}{|l|}{ YFP-16 } & \multicolumn{2}{|l|}{ GIN } \\
\hline & VGluT2+ & GlyT2+ & GlyT2- & GlyT2 + \\
\hline AP width (ms) & $0.52 \pm 0.09$ & $0.53 \pm 0.08$ & $0.78 \pm 0.13$ & $0.73 \pm 0.13$ \\
\hline AP half-width (ms) & $0.20 \pm 0.04$ & $0.21 \pm 0.04$ & $0.33 \pm 0.08$ & $0.32 \pm 0.07$ \\
\hline $\operatorname{AHP}(m V)$ & $19.9 \pm 2.8$ & $20.2 \pm 2.0$ & $27.0 \pm 3.0^{*}$ & $23.8 \pm 4.4^{*}$ \\
\hline Maximum derivative $(\mathrm{V} / \mathrm{s})$ & $536 \pm 117$ & $494 \pm 132$ & $341 \pm 70$ & $340 \pm 93$ \\
\hline Minimum derivative (V/s) & $472 \pm 104$ & $445 \pm 136$ & $255 \pm 70$ & $259 \pm 94$ \\
\hline $\operatorname{ADP}(V / s)$ & $7.54 \pm 5.58$ & $5.3 \pm 3.32$ & $-2.09 \pm 1.86$ & $-1.48 \pm 2.51$ \\
\hline Threshold (mV) & $-50.9 \pm 2.6$ & $-50.1 \pm 2.2$ & $\mathrm{n} / \mathrm{a}$ & $\mathrm{n} / \mathrm{a}$ \\
\hline Input resistance (MS ) & $46 \pm 19^{*}$ & $67 \pm 19^{*}$ & $248 \pm 136$ & $230 \pm 127$ \\
\hline Maximum firing rate $(\mathrm{Hz})$ & $398 \pm 136$ & $355 \pm 82$ & $145 \pm 32$ & $169 \pm 68$ \\
\hline Adaptation ratio@40 Hz & $0.72 \pm 0.12$ & $0.79 \pm 0.08$ & $0.91 \pm 0.08$ & $0.92 \pm 0.09$ \\
\hline Gain < $80 \mathrm{~Hz}(\mathrm{~Hz} / \mathrm{nA})$ & $178 \pm 63$ & $222 \pm 74$ & $240 \pm 71$ & $239 \pm 120$ \\
\hline Gain > $80 \mathrm{~Hz}(\mathrm{~Hz} / \mathrm{nA})$ & $98 \pm 37$ & $134 \pm 37$ & $202 \pm 64$ & $185 \pm 99$ \\
\hline Gain/input resistance & $2.27 \pm 0.74$ & $2.06 \pm 0.44$ & $0.97 \pm 0.39$ & $1.12 \pm 0.44$ \\
\hline Rebound firing (Hz) & $52 \pm 32^{*}$ & $21 \pm 16^{*}$ & $11 \pm 8$ & $8 \pm 7$ \\
\hline
\end{tabular}

Left columns indicate the physiology of YFP-16 neurons subjected to RT-PCR. Spike parameters, $n=9$ (each group); cell parameters, $n=7-9$. Right column indicate the physiology of GIN neurons that underwent RT-PCR. Most neurons expressed both GAD67 and GAD65; the data here are divided into those neuron that did or did not coexpress GlyT2 (spike parameters, $n=14$, each group; cell parameters, $n=11-14$ ). Data represent a separate pool of neurons than those analyzed in Table 2. Asterisks indicate $p<0.05$ compared to neurons from the same transgenic line. Values indicate mean \pm SD.

\section{A}

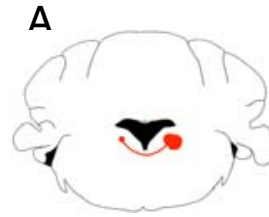

B
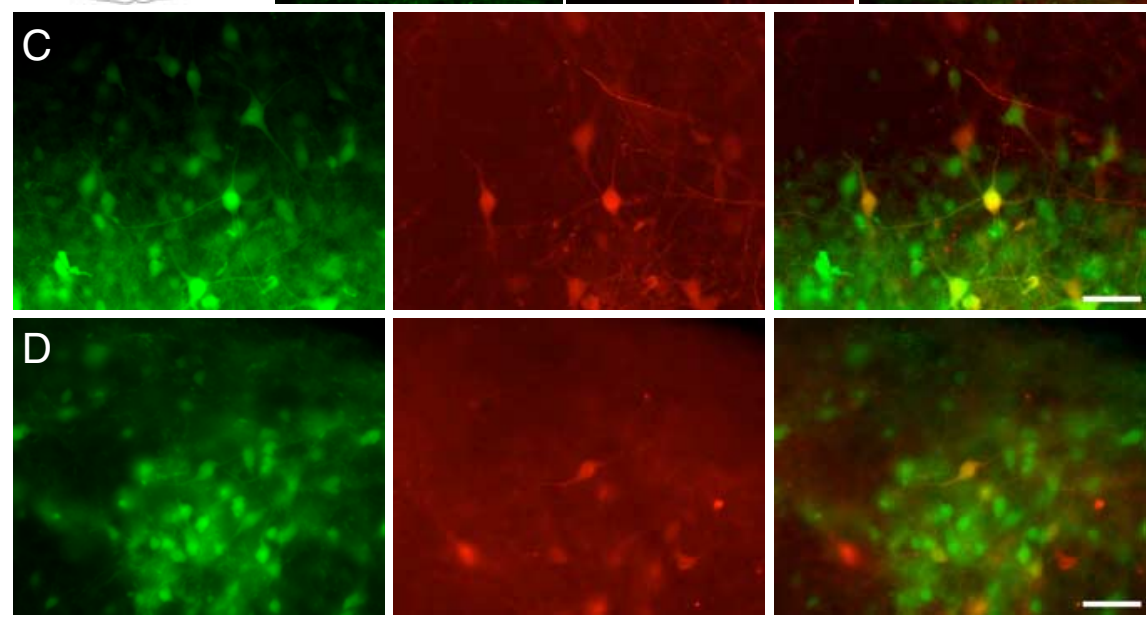

Figure 6. Identification of MVN neurons that project commissurally. A, Diagram of dye-labeling method. Crystals of fluorescently labeled dextran (fluororuby or Texas Red) were deposited unilaterally into the ventral aspect of the MVN in live slices. The dye was taken up by cut axons and transported retrogradely to cell bodies of origin. $\boldsymbol{B}$, Section from a GIN animal, showing axons at the midline. Left, GFP; middle, fluororuby; right, merge. Scale bar, $100 \mu \mathrm{m}$. C, D, Commissurally projecting YFP-16 (C) and GIN (D) neurons in the MVN contralateral to the injection. Scale bars, $50 \mu \mathrm{m}$.

the fluorescent labeling in a slice in which the unilateral MVN had been injected $\sim 6 \mathrm{~h}$ previously: on the left is the GFP signal of the fluorescent neurons, in the middle is the fluororuby signal, and on the right is the overlay. Double-labeled axons were seen coursing across the midline and entering or emerging from the medial longitudinal fasciculus (Fig. 6B) (GIN; also in YFP-16; data not shown). Figure $6 C$ shows examples of retrograde commissural label in the side of the MVN contralateral to dye injection in YFP-16 slices, whereas Figure $6 D$ shows the same for GIN slices. Double-labeled neurons were found in each line of mice, indicating that some YFP-16 and GIN neurons made projections to the contralateral MVN. Neurons were located both close to the ventricle and in somewhat more lateral portions of the MVN.

The physiology of those neurons that did project commissurally was examined in slices incubated for $>6 \mathrm{~h}$ at $\sim 32^{\circ} \mathrm{C}$ to allow for dye transport. Double-labeled Texas Red/YFP+ or fluororuby/GFP+ cells were easily identifiable under fluorescence and were targeted for recording. Figure $7 A$ shows example action potentials from a commissurally projecting YFP-16 neuron (left) and GIN neuron (right). Double-labeled neurons were analyzed, and the results were superimposed on data from previous Figures. Cellular characteristics of double-labeled YFP-16 and GIN neurons were highly similar to those of their respective parent populations, both in spike shape and in firing characteristics (Fig. $7 B, C$ ). Slight differences between a commissural population and the overall population may reflect age-dependent changes in neuronal physiology (Murphy and du Lac, 2001) because the commissural experiments were performed in somewhat younger animals (P14-P17). RT-PCR on a subset of commissurally labeled YFP-16 neurons indicated that all (6 of 6) were positive for GlyT2 but not VGluT2 $(p<0.05$ compared with the YFP-16 population as whole; Fisher's exact test). Intriguingly, commissurally projecting GIN neurons also frequently expressed GlyT2 (7 of 10). Therefore, commissural inhibition between the two sides of the MVN is accomplished by at least two highly distinct groups of neurons: GIN neurons, with a near-linear transformation of input and output that operates over relatively smaller ranges and may inhibit its targets with GABA and/or glycine, and glycinergic YFP-16 neurons, with a less linear but broader range of outputs.

\section{Schema for cell-type classification in the MVN}

It has been noted for several years that the intrinsic physiology of MVN neurons is heterogeneous and that two canonical types can be defined on the continuum of characteristics by their action potential waveforms and other traits (Serafin et al., 1991; Johnston et al., 1994). In this schema, action potential shapes have been grouped on the basis of the profile of the AHP: type A neurons, with monophasic AHPs, a "strong A-like rectification" (i.e., a slowed rate of rise in the AHP); type B, with biphasic AHPs; and sometimes type $\mathrm{C}$, a mixed group with anomalous characteristics. These groupings are surprisingly similar across species, including guinea pig, rat, and chick (Serafin et al., 1991; Johnston et al., 1994; du Lac and Lisberger, 1995).

Based on our recordings, GIN and YFP-16 neurons, respec- 
A
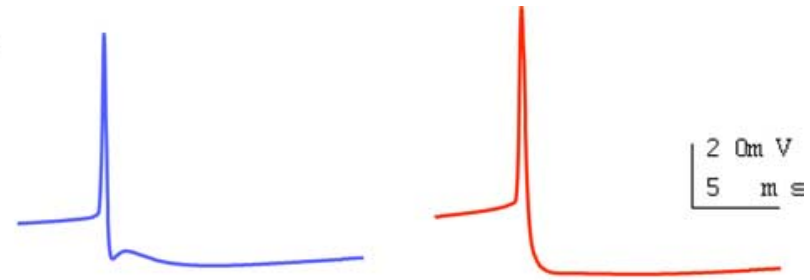

B
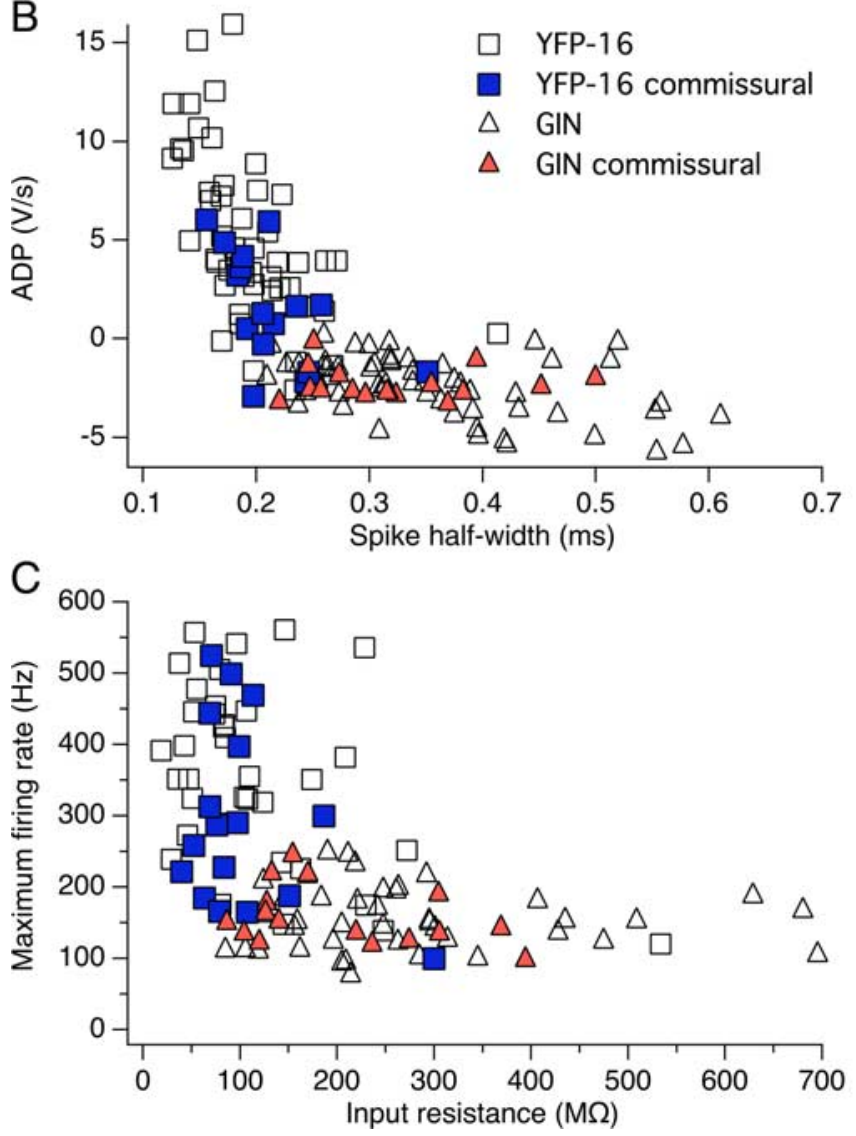

Figure 7. Physiology of identified commissural YFP-16 and GIN neurons. A, Action potentials from a YFP-16 commissural neuron (left, blue) and a GIN commissural neuron (right, red). $\boldsymbol{B}$, Data as in Figure 3C; the ADP is plotted versus the action potential half-width for each neuron. Commissural neurons in both lines display action potential waveforms similar to their parent populations [YFP-16 commissural (blue squares), $n=16$; GIN commissural (red triangles), $n=$ 17]. C, Reproduction of Figure 4C with data from commissural neurons overlaid (YFP-16, $n=$ 17; GIN, $n=16$ ). YFP-16 and GIN commissural neurons resemble the unidentified overall population of YFP-16 neurons. Slight differences may arise from developmental changes, because commissural recordings were made in younger animals (P14-P17 compared with P17-P28).

tively, appear to be excellent representatives of "type A" and "type B" groups, and, with the data presented above, we propose several criteria for sorting unidentified neurons into these two groups. We include data from GIN and YFP-16 neurons recorded at room temperature $\left(23^{\circ} \mathrm{C}\right)$, both to facilitate comparisons with data from other studies and to evaluate the resilience of our criteria to recording conditions (Table 2). The single most useful factor for dividing the populations is maximum firing rate, measured as the average rate across the highest depolarizing 1 s step over which a neuron can sustain continuous firing (Fig. 4). A dividing line at 220 spikes per second divides the populations at $34^{\circ} \mathrm{C}$ with $89 \%$ accuracy, quantified as number of neurons sorted correctly (74) divided by total number of neurons (83), and with $97 \%$ accuracy (33 of 34 ) in room temperature recordings. Although differences in the details of protocol such as species choice, electrode type, and internal/ external solution composition may alter the precise cutoff somewhat, its robustness to changes in temperature make this attribute a good candidate for analysis by other laboratories.

When data on neuronal maximum firing rate are unavailable, the measure of ADP defined here (the maximum value of the derivative during the $1 \mathrm{~ms}$ after the action potential repolarization to threshold) can be used both to divide the populations (Fig. $3 C$ ) and to provide an unbiased criterion for monophasic versus biphasic AHP. At $34^{\circ} \mathrm{C}$, an ADP cutoff value of $0 \mathrm{~V} / \mathrm{s}$ reliably classifies $94 \%$ of neurons (102 of 108). When spontaneous spikes from the neurons in this study were examined (as opposed to the spikes at a forced $10 \mathrm{~Hz}$ rate), the ADP was still 91\% (59 of 65) accurate at dividing the population, despite the fact that the spontaneous action potentials occurred at rates ranging from 3 to 70 $\mathrm{Hz}$. At $23^{\circ} \mathrm{C}$, however, only $83 \%$ of neurons ( 52 of 63 ) are classified correctly, suggesting that spike analysis of neurons at room temperature may be less reliable.

Although many other measures are different between the two populations of neurons (Table 2), the maximum firing rate (FR) and the ADP together are highly effective at dividing the population as follows:

$$
\begin{gathered}
\text { maximum FR }+25 \times \mathrm{ADP}<240 \rightarrow \mathrm{GIN}(\text { type } \mathrm{A}) \\
\text { maximum FR }+25 \times \mathrm{ADP}>240 \rightarrow \mathrm{YFP}-16(\text { type } \mathrm{B}) .
\end{gathered}
$$

This equation predicted the identity of $95 \%$ (79 of 83 ) of the neurons in this study at $34^{\circ} \mathrm{C}$ and $100 \%$ (34 of 34) of neurons at $23^{\circ} \mathrm{C}$. In addition, it correctly classified $96 \%$ ( 43 of 45 ) of neurons that underwent RT-PCR and were not included in the primary dataset used to derive this equation (Table 3 ). To verify the utility of the equation at identifying GABAergic neurons, we recorded from fluorescent neurons in the GAD67-GFP knock-in mouse, which carries EGFP in place of one of the native copies of the Gad1 gene. Ninety-two percent (22 of 24) of EGFP+ neurons were classified correctly as GIN-like, or GABAergic, by the above equation (Fig. 8). Therefore, our classification scheme may be generally useful to other laboratories attempting to distinguish classes of MVN neurons without the benefit of transgenic mouse lines.

\section{Discussion}

In this paper, we used transgenic mice with complementary subsets of fluorescently labeled neurons to supply a critical link between cell type and intrinsic physiology in the MVN. Glycinergic and glutamatergic neurons labeled in the YFP-16 mouse exhibit intrinsic membrane and firing properties distinct from those of GABAergic neurons in the GIN mouse, indicating that at least two information processing streams exist that differentially transform incoming signals to firing rate output. Surprisingly, glycinergic YFP-16 neurons display characteristics similar to glutamatergic rather than GABAergic neurons, suggesting that intrinsic physiological properties are matched to circuit function rather than to transmitter phenotype. Retrograde dye labeling indicates that both GABAergic and glycinergic neurons play a role in commissural signaling to the contralateral vestibular nuclei. Our results supply a schema for using action potential or firing properties to classify unidentified MVN neurons as GABAergic or non-GABAergic. 


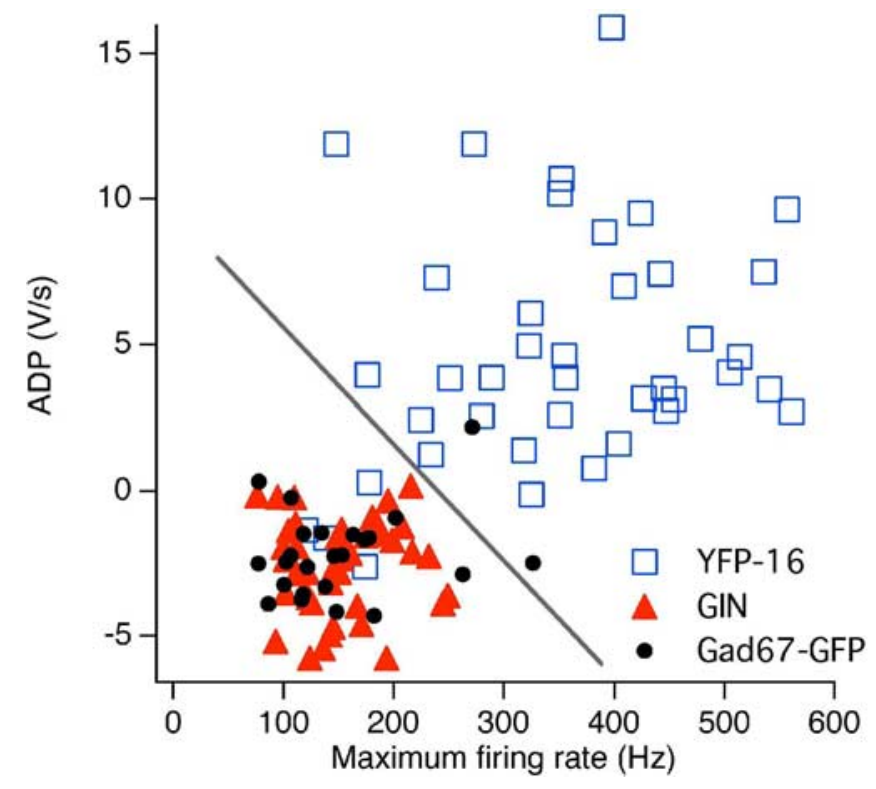

Figure 8. Measurements of intrinsic physiological characteristics successfully predict whether an MVN neuron is GABAergic. The action potential ADP value for each neuron is plotted versus its maximum firing rate; a line on the resulting graph accurately classifies $95 \%$ of YFP-16 and GIN neurons into their respective groups (see Results). The general utility of this dividing line was then evaluated by recording from GABAergic neurons labeled in the GAD67-GFP line, $92 \%$ of which are also classified correctly as GABAergic. YFP-16, $n=40$; GIN, $n=43$; GAD67$\mathrm{GFP}, n=24$.

\section{Cell-type classification in the MVN}

Intracellular recordings of MVN neurons in brain slices revealed that action potential properties vary continuously throughout the neuronal population (Serafin et al., 1991; Johnston et al., 1994; du Lac and Lisberger, 1995). Initial attempts to classify neurons in vitro subdivided the population into two types, A and $\mathrm{B}$, largely on the basis of the presence of second component to the AHP (Serafin et al., 1991; Johnston et al., 1994). Although action potentials characteristic of type A and type B neurons have been observed in various species (Straka et al., 2005), classification of neurons based on a continuous AHP profile requires semi-arbitrary subdivisions (Beraneck et al., 2003) and is not robust to changes in intrinsic physiological properties that occur with development (Dutia and Johnston, 1998; Murphy and du Lac, 2001), cellular plasticity (Nelson et al., 2003), or altered vestibular experience (Ris et al., 2002; Beraneck et al., 2003, 2004).

A significant advance in classifying MVN neurons in vitro used RT-PCR to identify the transmitter phenotype of physiologically characterized cells. Takazawa et al. (2004) showed that neurons with a monophasic AHP were primarily GABAergic and that glutamatergic neurons tended to exhibit an ADP. These results suggested that type A neurons were GABAergic but that neurons likely to be classified as type B based on their AHP profile could be either glutamatergic or GABAergic; glycinergic neurons were rarely detected.

Data from two distinct sets of MVN neurons identified in transgenic lines indicate that GABAergic neurons can be differentiated from glycinergic or glutamatergic neurons by using intrinsic physiological characteristics. Our results thus both extend and supplant previous classification schemes by providing objective criteria that subdivide MVN neurons into two types on the basis of maximum firing rate or $\mathrm{ADP}$, while taking advantage of the tools of mouse genetics to avoid relying on these measurements in future studies. Consistent with previous findings, GABAergic neurons, which encompass type A, have relatively wide action potentials (Takazawa et al., 2004); YFP-16 neurons exhibit narrow action potentials previously described in type $\mathrm{B}$ neurons (Serafin et al., 1991; Johnston et al., 1994). Both glutamatergic and glycinergic YFP-16 neurons (Table 3) exhibit firing properties characteristic of glutamatergic neurons recorded by Takazawa et al. (2004) as well as of presumed glutamatergic oculomotor nucleus projecting neurons (Sekirnjak and du Lac, 2006). The spatial segregation of GIN and YFP-16 neurons into the parvocellular and magnocellular divisions of the MVN, coupled with their transmitter phenotype, suggests that YFP-16 neurons project to ocular motor nuclei and spinal cord, whereas GIN neurons provide intrinsic connections within the vestibular complex (Epema et al., 1988; Buttner-Ennever, 1992).

Our classification scheme also illuminates previous results that relied on the type A/B division. Camp et al. (2006) reported that type B MVN neurons receive both glycinergic and GABAergic inputs, whereas type A receive only GABAergic inputs, suggesting that glutamatergic and glycinergic neurons are targets of glycinergic inputs, whereas GABAergic neurons are not. Unilateral labyrinthectomy differentially affects type A and type B neurons, triggering physiological changes that may cause one type to masquerade as the other (Him and Dutia, 2001; Beraneck et al., 2003, 2004). These results highlight the utility of the transgenic mice studied here for future studies.

\section{YFP-16 neurons in the MVN}

RT-PCR indicated that YFP-16 neurons targeted in this study express glycinergic or glutamatergic markers (Fig. 2). In vivo recordings and histology have shown that glycinergic neurons provide a major output of the MVN, namely that to the ipsilateral abducens nucleus, which controls the lateral rectus muscle (Spencer et al., 1989). We hypothesize that glycinergic YFP-16 neurons send axons to the abducens nucleus and/or the spinal cord, based on in vivo cell fills that show abducens-projecting MVN neurons also sending collaterals caudally (McCrea et al., 1980; Spencer et al., 1989). Glutamatergic neurons in the MVN are likely to make one of two major circuit projections: to motor neurons in the contralateral ocular motor nuclei or as mossy fibers to the cerebellar flocculus (Graf et al., 1997, 2002). Based on the physiological similarity between YFP-16 neurons and oculomotor-projecting neurons (Sekirnjak and du Lac, 2006), we propose that the glutamatergic YFP-16 neurons identified here are sources of input to the contralateral oculomotor nuclei and perhaps to the contralateral abducens. Glutamatergic floccularprojecting neurons in cats are located primarily in the dorsolateral, parvocellular MVN (Epema et al., 1990; Cheron et al., 1996), where YFP-16 neurons are sparse (Fig. $1 A$ ), arguing against the likelihood that YFP-16 neurons project to the flocculus. Further experiments will be needed to resolve this question.

\section{GABAergic neurons in the MVN}

Although GIN neurons comprise a subset of GAD67-expressing neurons (Oliva et al., 2000), their intrinsic physiological properties appear to be representative of GABAergic neurons more generally, as evidenced by recordings from GAD67-GFP knock-in mice (Fig. 8). GIN neurons in spinal cord and cortex have highly variable intrinsic and morphological attributes, suggesting that they may comprise several subgroups with different circuit roles (Heinke et al., 2004; Dougherty et al., 2005; Halabisky et al., 2006). GABAergic neurons in the MVN are thought to have five 
major targets: spinal motor areas, ipsilateral oculomotor and trochlear nuclei, inferior olive, ipsilateral MVN and prepositus neurons, and the contralateral MVN (Holstein, 2000). Because GIN neurons are found primarily in the parvocellular, intrinsically projecting region of the MVN (Fig. 1B) (Buttner-Ennever, 1992), and because their physiology is dissimilar to oculomotorprojecting neurons (Sekirnjak and du Lac, 2006), they are unlikely to fill the first two categories. Our data are consistent with the possibility that some GIN neurons project to the inferior olive (Gerrits et al., 1985; Barmack et al., 1993) and/or participate in feedforward inhibition from the vestibular nerve to other MVN neurons (Kinney et al., 1994; Straka and Dieringer, 1996). In addition, some GIN neurons project commissurally (Fig. 6); thus, they likely represent the source of contralateral GABAergic input that helps set up the push-pull dynamics of the VOR (Shimazu and Precht, 1966; Kasahara et al., 1968; Mano et al., 1968; Holstein et al., 1999).

Some GIN neurons, including those projecting commissurally, express GlyT2 (Fig. 2). GABA and glycine share the vesicular GABA transporter VGAT (Wojcik et al., 2006), and a subset of MVN neurons colocalizes GABAergic and glycinergic markers (Reichenberger et al., 1997; Takazawa et al., 2004; Tanaka and Ezure, 2004). Our data indicate that there are two types of glycinergic commissural neurons with distinct processing properties (Fig. $7 C, D$ ). It is possible that they target different populations of neurons in the contralateral MVN (Camp et al., 2006) and/or that the neurons colocalizing GABA and glycine appose postsynaptic neurons with selective receptor expression (Precht et al., 1973), as occurs in the vestibulocerebellum (Dugue et al., 2005).

\section{Physiological diversity of neuronal types}

Throughout the cortex and hippocampus, GABAergic interneurons are readily distinguished from glutamatergic pyramidal neurons by their narrow action potentials and fast firing rates (Cauli et al., 1997; Jonas et al., 2004). Surprisingly, we find that the reverse is true in the MVN. GABAergic neurons exhibit broader action potentials and slower maximum firing rates than those of putative projection neurons in the MVN. Because GABAergic and glycinergic neurons can display markedly different physiological characteristics while glutamatergic and glycinergic neurons exhibit similar ones, we propose that in vestibular circuits, cellular physiology is more closely related to the circuit role of a neuron rather than simply its neurotransmitters. The abducens and spinal cord, both of which are major components of vestibular circuits, receive glycinergic and glutamatergic input from the MVN. Thus the YFP-16 neurons, which are likely members of that circuit, share the responsibility of translating head motion into compensatory motor behavior and, as such, require a wide dynamic operating range. In contrast, GABAergic neurons, putatively local interneurons whose influence does not extend beyond the bilateral vestibular nuclei, exhibit a significantly narrower dynamic range. Although these intrinsic physiological attributes may reflect functional roles, they are labile and can change after experience (Straka et al., 2005; Gittis and du Lac, 2006). Thus the mouse lines described here should provide a natural springboard for studies of cell type-specific plasticity.

\section{References}

Attwell PJ, Cooke SF, Yeo CH (2002) Cerebellar function in consolidation of a motor memory. Neuron 34:1011-1020.

Autret L, Mechaly I, Scamps F, Valmier J, Lory P, Desmadryl G (2005) The involvement of Cav3.2/alpha1H T-type calcium channels in excitability of mouse embryonic primary vestibular neurones. J Physiol (Lond) 567:67-78.
Barmack NH, Fagerson M, Errico P (1993) Cholinergic projection to the dorsal cap of the inferior olive of the rat, rabbit, and monkey. J Comp Neurol 328:263-281.

Beraneck M, Hachemaoui M, Idoux E, Ris L, Uno A, Godaux E, Vidal PP, Moore LE, Vibert N (2003) Long-term plasticity of ipsilesional medial vestibular nucleus neurons after unilateral labyrinthectomy. J Neurophysiol 90:184-203.

Beraneck M, Idoux E, Uno A, Vidal PP, Moore LE, Vibert N (2004) Unilateral labyrinthectomy modifies the membrane properties of contralesional vestibular neurons. J Neurophysiol 92:1668-1684.

Bienhold H, Flohr H (1978) Role of commissural connexions between vestibular nuclei in compensation following unilateral labyrinthectomy [proceedings]. J Physiol (Lond) 284:178P.

Buttner-Ennever JA (1992) Patterns of connectivity in the vestibular nuclei. Ann NY Acad Sci 656:363-378.

Camp AJ, Callister RJ, Brichta AM (2006) Inhibitory synaptic transmission differs in mouse type $\mathrm{A}$ and $\mathrm{B}$ medial vestibular nucleus neurons in vitro. J Neurophysiol 95:3208-3218.

Cauli B, Audinat E, Lambolez B, Angulo MC, Ropert N, Tsuzuki K, Hestrin S, Rossier J (1997) Molecular and physiological diversity of cortical nonpyramidal cells. J Neurosci 17:3894-3906.

Cheron G, Escudero M, Godaux E (1996) Discharge properties of brain stem neurons projecting to the flocculus in the alert cat. I. Medical vestibular nucleus. J Neurophysiol 76:1759-1774.

De Zeeuw CI, Hansel C, Bian F, Koekkoek SK, van Alphen AM, Linden DJ, Oberdick J (1998) Expression of a protein kinase C inhibitor in Purkinje cells blocks cerebellar LTD and adaptation of the vestibulo-ocular reflex. Neuron 20:495-508.

Dieringer N, Precht W (1979a) Mechanisms of compensation for vestibular deficits in the frog. I. Modification of the excitatory commissural system. Exp Brain Res 36:311-328.

Dieringer N, Precht W (1979b) Mechanisms of compensation for vestibular deficits in the frog. II. Modification of the inhibitory pathways. Exp Brain Res 36:329-357.

Dougherty KJ, Sawchuk MA, Hochman S (2005) Properties of mouse spinal lamina I GABAergic interneurons. J Neurophysiol 94:3221-3227.

du Lac S, Lisberger SG (1995) Membrane and firing properties of avian medial vestibular nucleus neurons in vitro. J Comp Physiol A Neuroethol Sens Neural Behav Physiol 176:641-651.

du Lac S, Raymond JL, Sejnowski TJ, Lisberger SG (1995) Learning and memory in the vestibulo-ocular reflex. Annu Rev Neurosci 18:409-441.

Dugue GP, Dumoulin A, Triller A, Dieudonne S (2005) Target-dependent use of coreleased inhibitory transmitters at central synapses. J Neurosci 25:6490-6498.

Dutia MB, Johnston AR (1998) Development of action potentials and apamin-sensitive after-potentials in mouse vestibular nucleus neurones. Exp Brain Res 118:148-154.

Ebihara S, Yamamoto T, Obata K, Yanagawa Y (2004) Gene structure and alternative splicing of the mouse glycine transporter type-2. Biochem Biophys Res Commun 317:857-864.

Epema AH, Gerrits NM, Voogd J (1988) Commissural and intrinsic connections of the vestibular nuclei in the rabbit: a retrograde labeling study. Exp Brain Res 71:129-146.

Epema AH, Gerrits NM, Voogd J (1990) Secondary vestibulocerebellar projections to the flocculus and uvulo-nodular lobule of the rabbit: a study using HRP and double fluorescent tracer techniques. Exp Brain Res 80:72-82.

Faulstich BM, Onori KA, du Lac S (2004) Comparison of plasticity and development of mouse optokinetic and vestibulo-ocular reflexes suggests differential gain control mechanisms. Vision Res 44:3419-3427.

Feng G, Mellor RH, Bernstein M, Keller-Peck C, Nguyen QT, Wallace M, Nerbonne JM, Lichtman JW, Sanes JR (2000) Imaging neuronal subsets in transgenic mice expressing multiple spectral variants of GFP. Neuron 28:41-51.

Fremeau Jr RT, Troyer MD, Pahner I, Nygaard GO, Tran CH, Reimer RJ, Bellocchio EE, Fortin D, Storm-Mathisen J, Edwards RH (2001) The expression of vesicular glutamate transporters defines two classes of excitatory synapse. Neuron 31:247-260.

Fremeau Jr RT, Voglmaier S, Seal RP, Edwards RH (2004) VGLUTs define subsets of excitatory neurons and suggest novel roles for glutamate. Trends Neurosci 27:98-103. 
Furuya N, Yabe T, Koizumi T (1992) Neurotransmitters in the vestibular commissural system of the cat. Ann NY Acad Sci 656:594-601.

Gacek RR (1978) Location of commissural neurons in the vestibular nuclei of the cat. Exp Neurol 59:479-491.

Galiana HL, Flohr H, Jones GM (1984) A reevaluation of intervestibular nuclear coupling: its role in vestibular compensation. J Neurophysiol 51:242-259.

Gerrits NM, Voogd J, Magras IN (1985) Vestibular afferents of the inferior olive and the vestibulo-olivo-cerebellar climbing fiber pathway to the flocculus in the cat. Brain Res 332:325-336.

Gittis AH, du Lac S (2006) Intrinsic and synaptic plasticity in the vestibular system. Curr Opin Neurobiol 16:385-390.

Graf W, Spencer R, Baker H, Baker R (1997) Excitatory and inhibitory vestibular pathways to the extraocular motor nuclei in goldfish. J Neurophysiol 77:2765-2779.

Graf W, Gerrits N, Yatim-Dhiba N, Ugolini G (2002) Mapping the oculomotor system: the power of transneuronal labeling with rabies virus. Eur J Neurosci 15:1557-1562.

Halabisky B, Shen F, Huguenard JR, Prince DA (2006) Electrophysiological classification of somatostatin-positive interneurons in mouse sensorimotor cortex. J Neurophysiol 96:834-845.

Heinke B, Ruscheweyh R, Forsthuber L, Wunderbaldinger G, Sandkuhler J (2004) Physiological, neurochemical and morphological properties of a subgroup of GABAergic spinal lamina II neurones identified by expression of green fluorescent protein in mice. J Physiol (Lond) 560:249-266.

Highstein SM, Holstein GR (2005) The anatomy of the vestibular nuclei. Prog Brain Res 151:157-203.

Him A, Dutia MB (2001) Intrinsic excitability changes in vestibular nucleus neurons after unilateral deafferentiation. Brain Res 908:58-66.

Holstein GR (2000) Inhibitory amino acid transmitters in the vestibular nuclei. In: Neurochemistry of the vestibular system (Beitz AJ, Anderson JH, eds), pp 143-162. Boca Raton, FL: CRC.

Holstein GR, Martinelli GP, Cohen B (1999) The ultrastructure of GABAimmunoreactive vestibular commissural neurons related to velocity storage in the monkey. Neuroscience 93:171-181.

Johnston AR, MacLeod NK, Dutia MB (1994) Ionic conductances contributing to spike repolarization and after-potentials in rat medial vestibular nucleus neurones. J Physiol (Lond) 481:61-77.

Jonas P, Bischofberger J, Fricker D, Miles R (2004) Interneuron diversity series: fast in, fast out-temporal and spatial signal processing in hippocampal interneurons. Trends Neurosci 27:30-40.

Kasahara M, Mano N, Oshima T, Ozawa S, Shimazu H (1968) Contralateral short latency inhibition of central vestibular neurons in the horizontal canal system. Brain Res 8:376-378.

Kinney GA, Peterson BW, Slater NT (1994) The synaptic activation of $\mathrm{N}$-methyl-D-aspartate receptors in the rat medial vestibular nucleus. J Neurophysiol 72:1588-1595.

Ladpli R, Brodal A (1968) Experimental studies of commissural and reticular formation projections from the vestibular nuclei in the cat. Brain Res 8:65-96.

Lisberger SG, Pavelko TA, Broussard DM (1994) Neural basis for motor learning in the vestibuloocular reflex of primates. I. Changes in the responses of brain stem neurons. J Neurophysiol 72:928-953.

MacLean JN, Zhang Y, Johnson BR, Harris-Warrick RM (2003) Activityindependent homeostasis in rhythmically active neurons. Neuron 37:109-120.

Mano N, Oshima T, Shimazu H (1968) Inhibitory commissural fibers interconnecting the bilateral vestibular nuclei. Brain Res 8:378-382.

Marsicano G, Lutz B (1999) Expression of the cannabinoid receptor CB1 in distinct neuronal subpopulations in the adult mouse forebrain. Eur J Neurosci 11:4213-4225.

Mauk MD, Garcia KS, Medina JF, Steele PM (1998) Does cerebellar LTD mediate motor learning? Toward a resolution without a smoking gun. Neuron 20:359-362.

McCrea RA, Yoshida K, Berthoz A, Baker R (1980) Eye movement related activity and morphology of second order vestibular neurons terminating in the cat abducens nucleus. Exp Brain Res 40:468-473.

Medina JF, Nores WL, Ohyama T, Mauk MD (2000) Mechanisms of cerebellar learning suggested by eyelid conditioning. Curr Opin Neurobiol 10:717-724.

Metz AE, Jarsky T, Martina M, Spruston N (2005) R-type calcium channels contribute to afterdepolarization and bursting in hippocampal CA1 pyramidal neurons. J Neurosci 25:5763-5773.

Molineux ML, McRory JE, McKay BE, Hamid J, Mehaffey WH, Rehak R, Snutch TP, Zamponi GW, Turner RW (2006) Specific T-type calcium channel isoforms are associated with distinct burst phenotypes in deep cerebellar nuclear neurons. Proc Natl Acad Sci USA 103:5555-5560.

Murphy GJ, du Lac S (2001) Postnatal development of spike generation in rat medial vestibular nucleus neurons. J Neurophysiol 85:1899-1906.

Nelson AB, Krispel CM, Sekirnjak C, du Lac S (2003) Long-lasting increases in intrinsic excitability triggered by inhibition. Neuron 40:609-620.

Ohyama T, Nores WL, Medina JF, Riusech FA, Mauk MD (2006) Learning-induced plasticity in deep cerebellar nucleus. J Neurosci 26:12656-12663.

Oliva Jr AA, Jiang M, Lam T, Smith KL, Swann JW (2000) Novel hippocampal interneuronal subtypes identified using transgenic mice that express green fluorescent protein in GABAergic interneurons. J Neurosci 20:3354-3368.

Poyatos I, Ponce J, Aragon C, Gimenez C, Zafra F (1997) The glycine transporter GLYT2 is a reliable marker for glycine-immunoreactive neurons. Brain Res Mol Brain Res 49:63-70.

Precht W, Schwindt PC, Baker R (1973) Removal of vestibular commissural inhibition by antagonists of GABA and glycine. Brain Res 62:222-226.

Quadroni R, Knopfel T (1994) Compartmental models of type A and type B guinea pig medial vestibular neurons. J Neurophysiol 72:1911-1924.

Reichenberger I, Straka H, Ottersen OP, Streit P, Gerrits NM, Dieringer N (1997) Distribution of GABA, glycine, and glutamate immunoreactivities in the vestibular nuclear complex of the frog. J Comp Neurol 377:149-164.

Ris L, Hachemaoui M, Vibert N, Godaux E, Vidal PP, Moore LE (2001) Resonance of spike discharge modulation in neurons of the guinea pig medial vestibular nucleus. J Neurophysiol 86:703-716.

Ris L, Hachemaoui M, Godaux E (2002) Effect of labyrinthectomy on the spike generator of vestibular neurons in the guinea pig. NeuroReport 13:1875-1879.

Sekirnjak C, du Lac S (2002) Intrinsic firing dynamics of vestibular nucleus neurons. J Neurosci 22:2083-2095.

Sekirnjak C, du Lac S (2006) Physiological and anatomical properties of mouse medial vestibular nucleus neurons projecting to the oculomotor nucleus. J Neurophysiol 95:3012-3023.

Sekirnjak C, Vissel B, Bollinger J, Faulstich M, du Lac S (2003) Purkinje cell synapses target physiologically unique brainstem neurons. J Neurosci 23:6392-6398.

Serafin M, de Waele C, Khateb A, Vidal PP, Muhlethaler M (1991) Medial vestibular nucleus in the guinea-pig. I. Intrinsic membrane properties in brainstem slices. Exp Brain Res 84:417-425.

Shimazu H, Precht W (1966) Inhibition of central vestibular neurons from the contralateral labyrinth and its mediating pathway. J Neurophysiol 29:467-492.

Smith PF, Darlington CL, Curthoys IS (1986) Vestibular compensation without brainstem commissures in the guinea pig. Neurosci Lett 65:209-213.

Spencer RF, Wenthold RJ, Baker R (1989) Evidence for glycine as an inhibitory neurotransmitter of vestibular, reticular, and prepositus hypoglossi neurons that project to the cat abducens nucleus. J Neurosci 9:2718-2736.

Stahl JS, van Alphen AM, De Zeeuw CI (2000) A comparison of video and magnetic search coil recordings of mouse eye movements. J Neurosci Methods 99:101-110.

Straka H, Dieringer N (1996) Uncrossed disynaptic inhibition of secondorder vestibular neurons and its interaction with monosynaptic excitation from vestibular nerve afferent fibers in the frog. J Neurophysiol 76:3087-3101.

Straka H, Vibert N, Vidal PP, Moore LE, Dutia MB (2005) Intrinsic membrane properties of vertebrate vestibular neurons: function, development and plasticity. Prog Neurobiol 76:349-392.

Takazawa T, Saito Y, Tsuzuki K, Ozawa S (2004) Membrane and firing properties of glutamatergic and GABAergic neurons in the rat medial vestibular nucleus. J Neurophysiol 92:3106-3120. 
Tamamaki N, Yanagawa Y, Tomioka R, Miyazaki J, Obata K, Kaneko T (2003) Green fluorescent protein expression and colocalization with calretinin, parvalbumin, and somatostatin in the GAD67-GFP knock-in mouse. J Comp Neurol 467:60-79.

Tanaka I, Ezure K (2004) Overall distribution of GLYT2 mRNA-containing versus GAD67 mRNA-containing neurons and colocalization of both mRNAs in midbrain, pons, and cerebellum in rats. Neurosci Res 49:165-178

Varju P, Katarova Z, Madarasz E, Szabo G (2002) Sequential induction of embryonic and adult forms of glutamic acid decarboxylase during in vitro-induced neurogenesis in cloned neuroectodermal cell-line, NE7C2. J Neurochem 80:605-615.
Walsh MK, Lichtman JW (2003) In vivo time-lapse imaging of synaptic takeover associated with naturally occurring synapse elimination. Neuron 37:67-73.

Wojcik SM, Katsurabayashi S, Guillemin I, Friauf E, Rosenmund C, Brose N, Rhee JS (2006) A shared vesicular carrier allows synaptic corelease of GABA and glycine. Neuron 50:575-587.

Yue C, Remy S, Su H, Beck H, Yaari Y (2005) Proximal persistent $\mathrm{Na}^{+}$ channels drive spike afterdepolarizations and associated bursting in adult CA1 pyramidal cells. J Neurosci 25:9704-9720.

Zhang Q, Pangrsic T, Kreft M, Krzan M, Li N, Sul JY, Halassa M, Van Bockstaele E, Zorec R, Haydon PG (2004) Fusion-related release of glutamate from astrocytes. J Biol Chem 279:12724-12733. 